\title{
ANC From Experimental Perspective
}

\author{
Vaclav Burjan, Jaromir Mrazek* and Giuseppe D’Agata \\ Nuclear Physics Institute of the Czech Academy of Sciences, Prague, Czechia
}

In this article, we review the activities of the application of the Asymptotic Normalization Coefficients (ANC) method for the determination of the cross-sections and astrophysical $S$-factors of the radiative $(p, \gamma)$ captures, on stable and radioactive nuclei. A number of experiments were conducted at the Nuclear Physics Institute of the Czech Academy of Sciences, in cooperation with Texas A\&M University and the Istituto Nazionale di Fisica Nucleare - Laboratori Nazionali del Sud (Catania, Italy). These measurements were performed using solid state detectors and a magnetic spectrometer. This method was introduced in the last decade of the twentieth century and was, at first, used to contribute to the intensively studied topic of solar neutrinos. Later its use was extended from the $\mathrm{Li}, \mathrm{Be}$, and $\mathrm{B}$ element region to the $\mathrm{CNO}$ cycle and above. The obtained results were found (where other measurements were available) to be compatible with other indirect methods and even with direct measurements. While the capacities of

OPEN ACCESS

Edited by:

Scilla Degl'Innocenti,

University of Pisa, Italy

Reviewed by:

Pierre Descouvemont,

Université libre de Bruxelles, Belgium Andrea Celentano,

Universities and Research, Italy Juan Pablo Fernández García, Universidad de Sevilla, Spain

*Correspondence: Jaromir Mrazek mrazek@ujf.cas.cz

Specialty section: This article was submitted to Nuclear Physics,

a section of the journal Frontiers in Astronomy and Space

Sciences

Received: 15 May 2020

Accepted: 25 August 2020 Published: 10 November 2020

Citation:

Burjan V, Mrazek J and D'Agata G (2020) ANC From Experimental Perspective.

Front. Astron. Space Sci. 7:562466 doi: $10.3389 /$ fspas.2020.562466 direct measurements constantly improve, the advantage of the ANC and other indirect methods in general is still crucial in determining the astrophysical S-factors where short living isotopes participate, e.g., in ${ }^{11} \mathrm{C}(\mathrm{p}, \gamma){ }^{12} \mathrm{~N},{ }^{12} \mathrm{~N}(\mathrm{p}, \gamma){ }^{13} \mathrm{O}$, and ${ }^{13} \mathrm{~N}(\mathrm{p}, \gamma)^{14} \mathrm{O}$. The ANC method can also provide predictions for reactions with mirror nuclei. Other uses of ANCs are also discussed.

Keywords: nuclear physics, direct reactions, asymptotic normalization coefficient, astrophysical S-factor, CNO-cycle

\section{INTRODUCTION}

One of the important tasks of nuclear astrophysics is the precise determination of cross-sections of nuclear reactions. This knowledge enables determining reaction rates, which influence or determine the evolution of complex systems in astrophysical scenarios. When we consider quiescent (non-explosive) processes-their typical location is a star interior and the temperatures correspond to energies only about tens of $\mathrm{keV}$. Radiative captures-such as $(\mathrm{p}, \gamma),(\mathrm{n}, \gamma)$, $(\alpha, \gamma)$-typically appear in such environments.

Direct measurements in laboratory conditions at such low energies pose a great technical challenge and the analyses may still require the use of extrapolations to lower energies. The crosssections are strongly reduced due to the presence of the Coulomb barrier (units of $\mathrm{MeV}$ ) in case of charged particles. Neutron radiative capture measurements depend on the availability of the neutron beams at suitable energies and targets. Measurements with radioactive nuclei create another technological challenge. Indirect methods present useful tools, which do not completely replace the direct measurements, but they can add important pieces of independent information to deduce the desired cross-sections at energy regions of interest.

Nowadays, two indirect methods are used for radiative captures. (1) The Coulomb dissociation method (Baur et al., 1986) uses the reverse process of the radiative capture-the photodissociation reaction. The radiative capture process cross-section is then deduced from the detailed balance 
principle. The experimental feasibility of this method depends on a combination of several factors (see more in Baur and Rebel, 1996). (2) The other method is based on Asymptotic Normalization Coefficients.

\subsection{ANC}

The physics foundation of the method stems from the consideration that the direct radiative captures at low energies, proceed at large distances. Their cross-sections thus depend mostly on wave function tails, which have a known behavior and their amplitudes are given by a normalization coefficient. The Asymptotic normalization coefficients (ANC) method was developed in the last decade of the twentieth century (Xu et al., 1994). This method was used, in particular, to study the nuclear processes in stars (the p-p chain, CNO cycles, and cycles with heavier elements) and to investigate the Big Bang Nucleosynthesis (BBN). The interactions in these processes predominantly have a surface character. The peripheral character is also typical for direct nuclear reactions (DR), that are thus, predetermined as a tool to study the radiative captures. It appears that cross-sections of direct transfer reactions contain the same elements-vertex constants-that determine the direct radiative capture. These elements are closely related to the amplitudes of asymptotic tails of the (radial overlap) wave functions of the participating nuclei. These amplitudes are called asymptotic normalization coefficients (ANC).

The ANC method provides an accurate tool to determine the direct capture cross-section using experimental information from peripheral nucleon transfers.

\subsection{Strengths and Weaknesses of ANC}

Transfer reactions can be studied at larger energies than those typical for nucleosynthesis and even with radioactive beams. Their cross-sections are usually orders of a magnitude larger than direct radiative captures at astrophysically low energies.

Uncertainties of the ANC method are related to the well-known model dependence of Distorted Wave Born Approximation (DWBA) calculations: on the choice of the optical model potential (OMP) and the transferred single particle wave function. By choosing appropriate reactions and beam energies, the peripheral mechanism can be selected and thus the uncertainty associated with the choice of optical model potentials can be minimized.

The application of the ANC method in nuclear astrophysics is based on these assumptions:

- The final state is weakly bound

- The absence of resonances

Under these conditions, the direct radiative capture cross section is crucial [e.g., ${ }^{7} \mathrm{Be}(\mathrm{p}, \gamma){ }^{8} \mathrm{~B}$ ]. States present around the threshold (at higher masses, larger binding energies) render the resonant capture more dominant. The contribution of the direct capture may fall orders of a magnitude below the total capture.

Nevertheless, the ANCs can

- Play an important role, where resonances are narrow and distant from the energy region of interest,
- Influence the interference of resonant contributions $\left({ }^{15} \mathrm{~N}(\mathrm{p}, \gamma){ }^{16} \mathrm{O}\right)$

- Still play a significant role in the cross-section at low energies in case of a sub-threshold resonance $\left({ }^{20} \mathrm{Ne}(\mathrm{p}, \gamma){ }^{21} \mathrm{Na}\right)$,

- Be used to deduce the width of the resonance in the mirror nucleus $\left({ }^{26} \mathrm{Mg}(\mathrm{n}, \gamma){ }^{27} \mathrm{Mg}\right)$.

After each reaction, we assess the applicability of the ANC method.

\subsection{Paper Organization}

The paper is organized into five sections:

- Section 1 - is the introduction,

- In section 2, we describe principles of the ANC method,

- In section 3, selected reactions with ANC application are presented,

- In section 4, ANC applications for mirror nuclei and nuclear radii are briefly described,

- Section 5 - we provide a summary.

\subsection{Aim of the Paper}

Several reviews concerning ANC were recently published:

Huang et al. (2010) presented calculated ANC values within the simplified two-body model and consideration of singleparticle states. Comparison to available experimental values works well for many cases.

Timofeyuk (2013) used a source term approach (STA) with shell model wave functions to calculate ANCs within a $0-p$ shell. It is an update of the previous review of Timofeyuk (2010).

The aim of the paper is to review the experimental cases, where the authors have a deeper experimental insight, as they were part of the experimental team. The selection of reactions follows a chronological order, to show connections (where present) between experiments.

\section{THE METHOD OF ASYMPTOTIC NORMALIZATION COEFFICIENTS}

In case of low energy reactions of astrophysical interest, we usually express the cross-section for charged particles by the astrophysical S-factor to eliminate the Coulomb dependence of the cross-section at low energies. Without loss of generality we can consider a proton radiative capture

$$
A+p \rightarrow B+\gamma
$$

Then the astrophysical S-factor is expressed as

$$
S(E)=E e^{2 \pi \eta} \sigma(E),
$$

where $\mathrm{E}$ is the center of mass energy of the relative motion of the nucleus $A$ and proton, $\eta$ is the Sommerfeld parameter $\eta=Z_{A} Z_{p} e^{2} / \hbar v\left(Z_{A}\right.$ being the charge of the nucleus $A, Z_{p}=1$ and $v$ the relative velocity) and $\sigma(E)$ is proportional to the square of the transition matrix element for the direct capture (see e.g., Xu et al., 1994):

$$
\begin{aligned}
\sigma(E) \approx & \left|\left\langle\psi_{B}\left|\hat{O}^{(X L)}\right| \psi_{A} \psi_{p} \chi^{(+)}\right\rangle\right|^{2} \\
& \approx\left|\left\langle I_{A p}^{B}\left|\hat{O}^{(X L)}\right| \chi^{(+)}\right\rangle\right|^{2},
\end{aligned}
$$


where $\psi_{A}$ and $\psi_{p}$ are the wave functions of the nucleus $\mathrm{A}$ and proton, $\chi_{A}^{(+)}$is the distorted wave function in the entrance channel, $\psi_{B}$ is the wave function of the nucleus $\mathrm{B}$, and $\hat{O}^{(X L)}$ the electromagnetic operator, where $(X L)$ refers to the electric or magnetic multipole, frequently $E 1$. The overlap integral $\mathrm{I}_{A p}^{B}(\vec{r})$ between the initial and final state can be written as:

$$
\begin{gathered}
\mathrm{I}_{A p}^{B}(\vec{r})=\sum_{l_{B} m_{l_{B} j_{B} m_{j_{B}}}} i^{l_{B}}\left\langle J_{A} M_{A} J_{p} M_{p} \mid j_{B} m_{j_{B}}\right\rangle\left\langle j_{B} m_{j_{B}} l_{B} m_{B}\right| \\
\left.J_{B} M_{B}\right\rangle \times I_{A p l_{B} j_{B}}^{B}(r) Y_{l_{B} m_{l_{B}}}(\vec{r}) .
\end{gathered}
$$

The radial part of the overlap function can be approximated by a model wave function $\phi_{n l j}(r)$ of the proton bound state $B=(A p)$

$$
I_{A p l_{B} j_{B}}^{B}(r)=S_{A p l_{B} j_{B}}^{1 / 2} \phi_{n_{B} l_{B} j_{B}}(r)
$$

where $S$ is the spectroscopic factor.

The tail of the nuclear overlap function contributes to the matrix element, especially at low energies. The shape of this tail has a well-known asymptotic behavior and its amplitude alone gives the rate of the direct part of the capture reaction. The asymptotic behavior of the radial overlap function is expressed by the Whittaker function W (see Mukhamedzhanov et al., 1997)

$$
I_{A p}^{B}\left(r_{A p}\right) \stackrel{r_{A p}>R_{B}}{=} C_{A p}^{B} \frac{W_{l_{A}+1 / 2}\left(2 k_{A p} r_{A p}\right)}{r_{A p}}
$$

Here, the asymptotic normalization coefficient $C_{A p}^{B}$ for the system $A+p \rightarrow B$ specifies the amplitude of the proton tail of the wave function for nucleus $B$, for distances larger than the nuclear radius $R_{B}$ and $k$ is the wave number. This normalization coefficient determines the corresponding direct capture cross-section.

The asymptotic behavior of the model wave function $\phi_{n_{B} l_{B} j_{B}}(r)$ of the proton bound state can be written as

$$
\phi_{n_{B} l_{B} j_{B}}\left(r_{A p}\right) \stackrel{r_{A p}>R_{B}}{=} b_{A p} \frac{W_{l_{A}+1 / 2}\left(2 k_{A p} r_{A p}\right)}{r_{A p}} .
$$

The quantity $b_{A p}$ represents the single-particle ANC (SPANC) and defines the amplitude of the tail of the radial single-particle bound-state wave function.

For peripheral reactions, the differential cross-section calculated in DWBA approximation can be replaced by an integral over the external region, where the bound state is replaced by the Whittaker function, multiplied by the ANC. The cross-section of the direct $(p, \gamma)$ can be finally expressed using ANC and SPANC from Equations (3) and (4) as in (Bertulani, 2003):

$$
\sigma(E)=\sum \frac{\left(C_{A p l_{B} j_{B}}^{B}\right)^{2}}{\left(b_{A p l_{B} j_{B}}\right)^{2}} \sigma_{l_{B} j_{B}}^{(\mathrm{cap})}(E)
$$

where $\sigma_{l_{B} j_{B}}^{(\text {cap })}(E)$ is the cross-section for the electromagnetic transition to the final state $l_{B} j_{B}$.

The same asymptotic normalization coefficient $C_{A p}^{B}$ as that in the radiative capture can be obtained from the direct transfer reactions. In the DWBA theory for the $\mathrm{A}(a, b) \mathrm{B}$ reaction, where $a=p+b, \mathrm{~B}=\mathrm{A}+p$, and $p$ being the transferred proton, the transition amplitude is

$$
M=\sum_{M_{a}}\left\langle\chi_{f}^{(-)} \psi_{B} \varphi_{b}|\Delta V| \varphi_{a} \psi_{A} \chi_{i}^{(+)}\right\rangle,
$$

where $\chi_{i}^{(+)}, \chi_{f}^{(-)}$are distorted wave functions in input and output channels, $\psi_{A}, \psi_{B}$ are wave functions describing inner states of A, B nuclei, $\varphi_{a}, \varphi_{b}$ are wave functions of particles $a$, and $b$. The transition operator $\Delta V$ has a shape $V_{b p}+U_{b A}-U_{\beta}$ for post form, where $U_{\beta}$ is the optical potential in final channel. The transition amplitude $M$ can be expressed as

$$
M=\left\langle\chi_{f}^{(-)} I_{A p}^{B}|\Delta V| I_{b p}^{a} \chi_{i}^{(+)}\right\rangle,
$$

where $I_{A p}^{B}=S^{1 / 2} \phi_{n l j}\left(r_{A p}\right)$ is the above mentioned overlap integral and $I_{p b}^{a}$ is the overlap integral of $a$ and $b$ particles, in analogy with $I_{A p}^{B}$. The experimental cross-section for the transfer proton reaction can be expressed at the asymptotic region larger than the nuclear radius $R_{B}$ in this way (Mukhamedzhanov et al., 1997)

$$
\frac{d \sigma(\theta)}{d \Omega}=\sum \frac{\left(C_{A p l_{B} j_{B}}^{B}\right)^{2}\left(C_{p b l_{a} j_{a}}^{a}\right)^{2}}{\left(b_{A p l_{B} j_{B}}\right)^{2}\left(b_{p b l_{a j} j_{a}}\right)^{2}} \sigma_{l_{B j_{B} l_{a} j_{a}}^{D W}}(\theta)
$$

Here, $\left(C_{A p l_{B} j_{B}}^{B}\right)^{2}$ and $\left(C_{p b l_{a j} j_{a}}^{a}\right)^{2}$ are ANCs of the systems $A+p$ and $p+b, j_{i}$ and $l_{i}$ are the total and orbital angular momenta of the transferred proton, respectively. Coefficients $b$ are the singleparticle ANCs, defining the amplitude of the tail of the radial proton bound-state wave function. $\sigma_{l_{l_{B} j_{B} l_{a j a}}^{D W}}(\theta)$ is the deduced DWBA cross-section. Using the above expression, it is possible to express the cross-section of the direct proton capture at very low energies by the relation (5) where the normalization factor $\left(C_{A p l_{B} j_{B}}^{B}\right)^{2}$ is known from the transfer reaction $(a, b)$.

For peripheral reaction, where only the outer region of the nuclear radial integrals contributes to the cross-section, the ratio

$$
R\left(b_{A p l_{B} j_{B}}, b_{p b l_{a} j_{a}}\right)=\frac{\sigma_{l_{B} j_{B} l_{a j_{a}}}^{D W(\max )}(\theta)}{\left(b_{A p l_{B} j_{B}}\right)^{2}\left(b_{p b l_{a j_{a}}}\right)^{2}},
$$

should not depend on the single particle ANCs $b_{A p}$ and $b_{b p}$. The $\sigma^{D W(\max )}(\theta)$ is the DWBA differential cross-section at the main maximum of the angular distribution.

The stability of this ratio represents an important peripherality check of the transfer reaction. 


\section{SELECTED EXPERIMENTAL CASES}

In the following section, we introduce several experimental cases, where the ANCs were determined.

At first, we illustrate in detail the experimental investigation of the direct capture of ${ }^{7} \mathrm{Be}(\mathrm{p}, \gamma)^{8} \mathrm{~B}$. This reaction is in the center of the so-called solar neutrino problem. There was a discrepancy between the prediction of the high energy solar neutrino flux and the measured values (Bahcall, 1985). The measured values of the neutrino flux represented one third of the predicted flux. These neutrinos are mainly products of the decaying ${ }^{8} \mathrm{~B}$ from the radiative capture ${ }^{7} \mathrm{Be}(\mathrm{p}, \gamma)^{8} \mathrm{~B}$. The determination of astrophysical $S$-factor $S_{17}(0)$ for this capture seems to be the key to the problem.

Nowadays, the discrepancy between measured and produced flux of solar neutrinos is explained by neutrino oscillations (Ahmad et al., 2001; Bellerive et al., 2016-SNO collaboration) however, the independently and indirectly measured S-factor remains important for stellar models.

The suitable proton transfer reaction to study the direct capture ${ }^{7} \mathrm{Be}(\mathrm{p}, \gamma){ }^{8} \mathrm{~B}$ by ANC method would be ${ }^{8} \mathrm{~B}\left({ }^{7} \mathrm{Be},{ }^{8} \mathrm{~B}\right){ }^{7} \mathrm{Be}$. This reaction, due to the symmetry, contains the same ANC value twice and thus, the determination would not be affected by other ANCs. However, both reaction participants are radioactive nuclei, so, the other transfer reactions were selected: ${ }^{10} \mathrm{~B}\left({ }^{7} \mathrm{Be},{ }^{8} \mathrm{~B}\right){ }^{9} \mathrm{Be}$ and ${ }^{14} \mathrm{~N}\left({ }^{7} \mathrm{Be},{ }^{8} \mathrm{~B}\right){ }^{13} \mathrm{C}$. These reactions both contain two different ANC values. The ANCs for the virtual decay ${ }^{10} \mathrm{~B} \rightarrow{ }^{9} \mathrm{Be}+p$ and ${ }^{14} \mathrm{~N} \rightarrow{ }^{13} \mathrm{C}+p$ had to be deduced. The techniques are described in more detail for the first reaction, and the details will not be repeated in the following experiments.

\section{1. ${ }^{9} \mathrm{Be}(p, \gamma){ }^{10} \mathrm{~B}$}

The determination of ANC for the ${ }^{9} \mathrm{Be}(\mathrm{p}, \gamma){ }^{10} \mathrm{~B}$ radiative capture was one of the problems when the method of ANCs was first applied by Mukhamedzhanov et al. (1997). A suitable transfer reaction is ${ }^{9} \mathrm{Be}\left({ }^{10} \mathrm{~B},{ }^{9} \mathrm{Be}\right){ }^{10} \mathrm{~B}$, as it is symmetric in ANCs. At first, we measured the elastic scattering ${ }^{10} \mathrm{~B}+{ }^{9} \mathrm{Be} \rightarrow{ }^{10} \mathrm{~B}+{ }^{9} \mathrm{Be}$ to find the corresponding optical model parameters. Then, these parameters were used for the analysis of the proton transfer reaction ${ }^{9} \mathrm{Be}\left({ }^{10} \mathrm{~B},{ }^{9} \mathrm{Be}\right){ }^{10} \mathrm{~B}$. The measurement was performed at the Cyclotron Institute of the Texas A\&M University using $100 \mathrm{MeV}$ beam of ${ }^{10} \mathrm{~B} .{ }^{9} \mathrm{Be}$ targets with thicknesses between 200 and $300 \mu \mathrm{g} / \mathrm{cm}^{2}$ were prepared by evaporation. The targets were located in the focal plane of the Multipole magnetic spectrometer (MDM). The magnetic spectrometer was tuned to measure either elastically scattered ${ }^{10} \mathrm{~B}$ ions or ${ }^{9} \mathrm{Be}$ ions from the proton transfer reaction. The measurement of specific energy loss in the ionization chamber was used for particle identification and the residual energy was measured by a NE102A plastic scintillator located behind the exit window of the detector. The entrance and exit windows of the detector was made of 1.8 and $7.2 \mathrm{mg} / \mathrm{cm}^{2}$ thick Kapton foils, respectively. The ionization chamber was filled with pure isobutane at a pressure of $30 \mathrm{mbar}$. Outgoing ${ }^{9} \mathrm{Be}$ nuclei at forward angles are kinematically equivalent to ${ }^{10} \mathrm{~B}$ elastically scattered in the backward hemisphere. The experimental elastic scattering was fitted with three optical model potentials, that differed mainly in depths of the real part of the OM potential (Mukhamedzhanov et al., 1997, see in Figure 1). Resulting fits

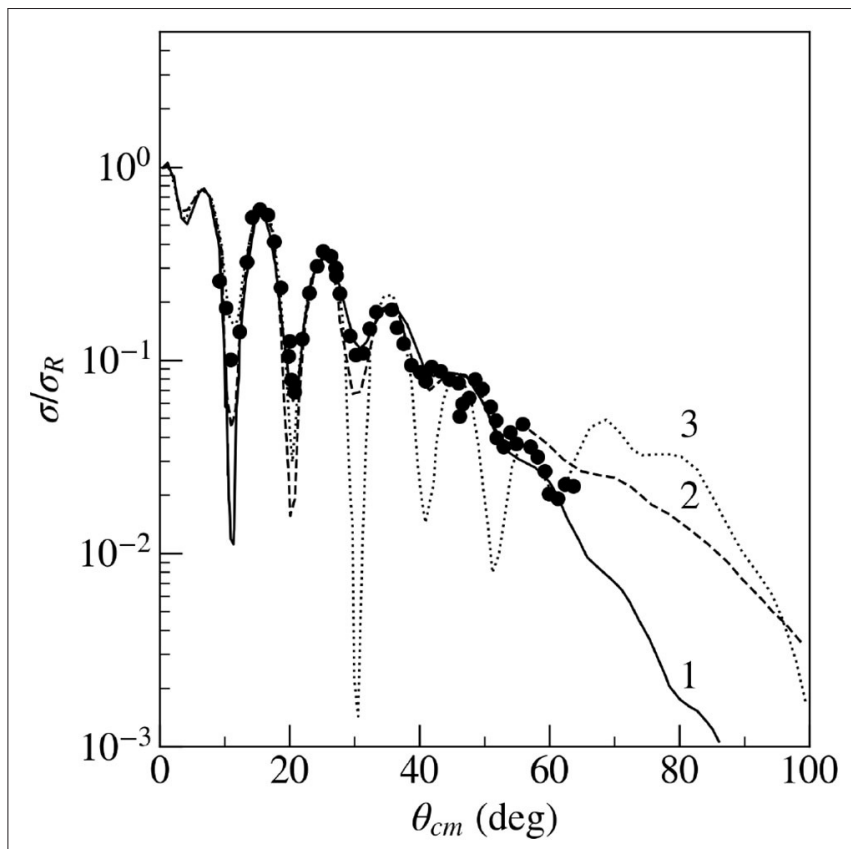

FIGURE 1 | The elastic scattering cross-section of the ${ }^{10} \mathrm{~B}$ ions on the ${ }^{9} \mathrm{Be}$ target, fitted by optical model calculations with potentials 1 -solid line, potential 2-dashed line, and potential 3-dotted line, the depths of the real part of these three optical model potentials were 64.2, 131.2, and $203.2 \mathrm{MeV}$, respectively, see details in Mukhamedzhanov et al. (1997). Ratio to Rutherford scattering is on the $y$-axis.

are plotted in Figure 1. The potential 3 gave substantially worse $\chi^{2}$ and therefore, it was rejected for further analysis. With these optical model parameters, the angular distribution for the proton transfers to different final states of the ${ }^{10} \mathrm{~B}$ were calculated by means of the PTOLEMY (Rhoades-Brown et al., 1983) code. The example of the fitted experimental angular distribution of the proton transfer to the ground state of ${ }^{10} \mathrm{~B}$ is given in Figure 2 . ANCs for the proton transfer reaction from the calculation with parameters of the optical models 1 and 2 are given in the Table 1. Several tests were made to prove the applicability of the ANC method. The peripheral character of the ${ }^{9} \mathrm{Be}\left({ }^{10} \mathrm{~B},{ }^{9} \mathrm{Be}\right){ }^{10} \mathrm{~B}$ reaction was verified. The ANC method is applicable, when the transfer process is peripheral. The peripherality can be demonstrated by showing that the cross-section does not depend on the inner part of the reaction region. Calculation of DWBA angular distribution with different cut-off radii did not reveal significant variations, when using values lower that $5 \mathrm{fm}$. This allows replacing the bound state function with the Whittaker asymptotic form. A next check was performed for the $R$ function (Equation 9). This function should be constant in a region (1.1 $\leq r_{0} \leq 1.3 \mathrm{fm}$ and $0.5 \leq a \leq 0.7 \mathrm{fm}$ ), where $r_{0}$ and $a$ are bound state potential well parameters. Then the dependence on the selection of a potential is weak. In the presented case, these differences are small (see Table 1, where they are included in uncertainties of ANCs). By changing parameters $r_{0}$ and $a$ it is possible to demonstrate that the dependence of the spectroscopic factor on the single particle ANC $b_{l j}$ is strong, it varies by factor 


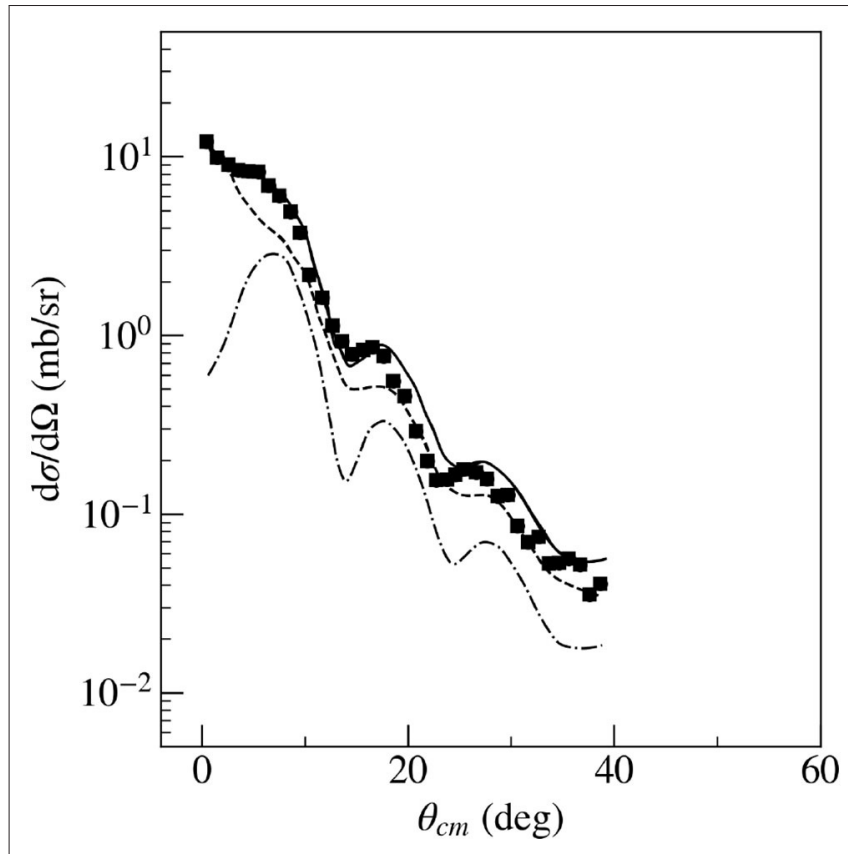

FIGURE 2 | The calculated angular distributions of the reaction ${ }^{9} \mathrm{Be}\left({ }^{10} \mathrm{~B},{ }^{9} \mathrm{Be}\right){ }^{10} \mathrm{~B}$ (g.s.) compared with the experimental data (black points). The DWBA fits for individual contributions of $\mathrm{I}=0,1$, and 2 are represented by dashed, dotted, and dash-dotted lines, respectively. Calculations are performed with the optical potential 1 and the optical potential 2 gives the same fit.

TABLE 1 | The extracted $\mathrm{ANCs}\left(C^{2}\right)$ for the ${ }^{9} \mathrm{Be}(\mathrm{p}, \gamma){ }^{10} \mathrm{~B}$ capture from the ${ }^{9} \mathrm{Be}\left({ }^{10} \mathrm{~B},{ }^{9} \mathrm{Be}\right){ }^{10} \mathrm{~B}$ reaction.

\begin{tabular}{lcccc}
\hline $\boldsymbol{E}^{*}(\boldsymbol{M e V})$ & $\boldsymbol{j}_{\boldsymbol{p}}$ & $\boldsymbol{C}_{\mathbf{1}}^{\mathbf{2}}\left(\boldsymbol{f m}^{-\mathbf{1}}\right)$ & $\boldsymbol{C}_{\mathbf{2}}^{\mathbf{2}}\left(\mathbf{f m}^{-\mathbf{1}}\right)$ & $\boldsymbol{C}^{\mathbf{2}}\left(\mathbf{f m}^{-\mathbf{1}}\right)$ \\
\hline 0.0 & $3 / 2$ & $4.91(19)$ & $5.35(21)$ & $5.06(46)$ \\
0.718 & $1 / 2$ & $1.23(15)$ & $1.34(16)$ & $1.27(21)$ \\
& $3 / 2$ & $3.33(17)$ & $3.63(19)$ & $3.43(42)$ \\
1.740 & $3 / 2$ & $4.22(33)$ & $4.60(36)$ & $4.35(59)$ \\
2.154 & $1 / 2$ & $0.28(5)$ & $0.30(5)$ & $0.29(6)$ \\
& $3 / 2$ & $0.80(8)$ & $0.87(9)$ & $0.82(12)$
\end{tabular}

$C_{1}^{2}$ and $C_{2}^{2}$ are deduced ANCs using optical potentials 1 and 2, respectively, and the bound state potential with $r_{0}=1.20 \mathrm{fm}$ and $\mathrm{a}=0.60 \mathrm{fm}$. The third $C^{2}$ is the adopted average value and uncertainty.

3 (Figure 3), while $C^{2}$ changes in the considered region by only about $10 \%$.

The extracted ANCs from Table 1 were then used for the analysis of the proton transfer reaction ${ }^{10} \mathrm{~B}\left({ }^{7} \mathrm{Be},{ }^{8} \mathrm{~B}\right){ }^{9} \mathrm{Be}$ which is suitable in determining the normalization of the cross-section for the direct proton capture ${ }^{7} \mathrm{Be}(\mathrm{p}, \gamma){ }^{8} \mathrm{~B}$ at low astrophysical energies.

The ANC of ${ }^{9} \mathrm{Be}(p, \gamma){ }^{10} \mathrm{~B}$ reaction, due to the applicability conditions, was only used for the analysis of ${ }^{7} \mathrm{Be}(\mathrm{p}, \gamma)^{8} \mathrm{~B}$ capture.

\section{2. ${ }^{16} \mathrm{O}(p, \gamma){ }^{17} \mathrm{~F}$}

Prior to the application of ANC method on ${ }^{7} \mathrm{Be}(\mathrm{p}, \gamma){ }^{8} \mathrm{~B}$, the ANC method was tested on the ${ }^{16} \mathrm{O}(\mathrm{p}, \gamma){ }^{17} \mathrm{~F}$ capture. The

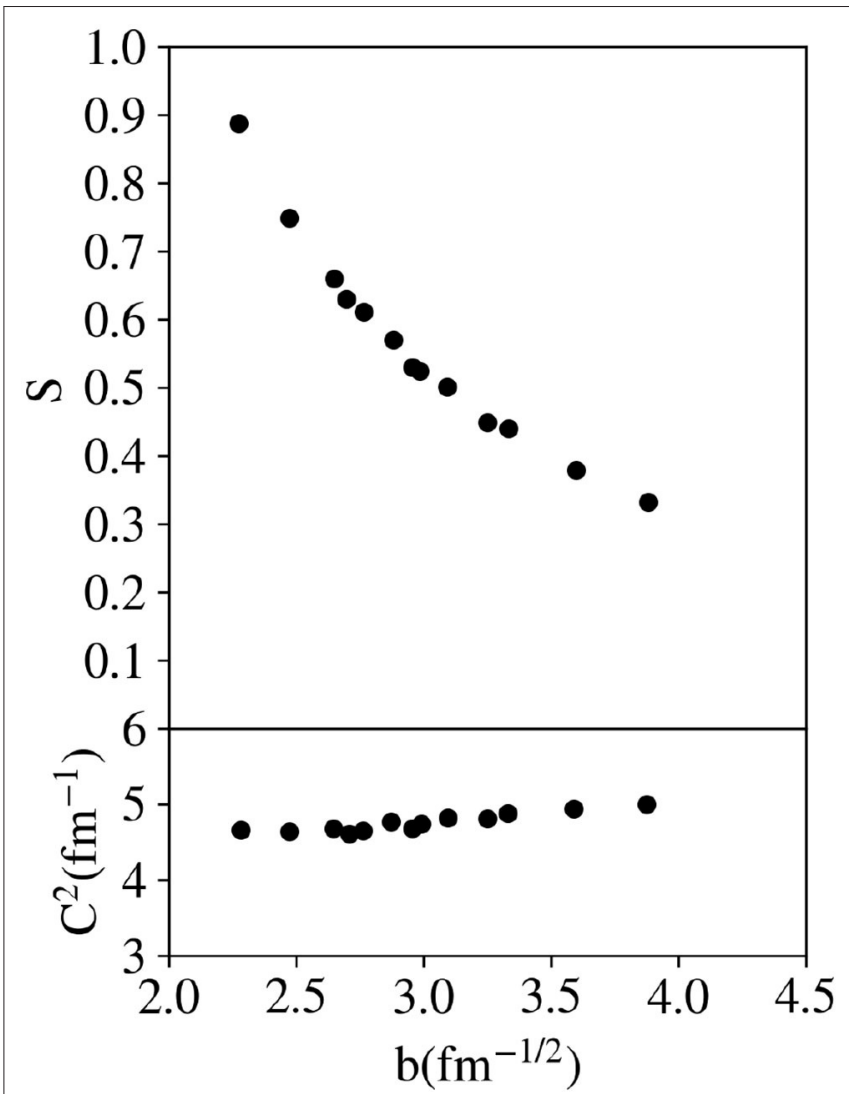

FIGURE 3 | The dependence of the spectroscopic factor (upper) and ANC $\left(C^{2}\right)$ (lower) on the geometry of the bound state potential of the state ${ }^{9} \mathrm{Be}\left(3 / 2^{-}\right)+p\left(j_{p}=3 / 2^{-}\right)$in ${ }^{10} \mathrm{~B}($ g.s. $)$. The geometry is expressed by the different single particle ANCs (value $b$, see Equation 4), i.e., achieved by changing the parameters $r_{0}$ and $a$ of the bound state potential in some intervals.

direct measurement data at low energies (Chow et al., 1975; Morlock et al., 1997) were available for comparison. Two separate measurements were performed for the transfer reaction ${ }^{16} \mathrm{O}\left({ }^{3} \mathrm{He}, \mathrm{d}\right){ }^{17} \mathrm{~F}$ (Gagliardi et al., 1999).

One measurement was performed at the Nuclear Physics Institute of the Czech Academy of Sciences, Řež, (NPI CAS), using a $27.7 \mathrm{MeV}{ }^{3} \mathrm{He}$ beam. The absolute differential crosssection for the process was measured between $6.5^{\circ}$ and $25^{\circ}$ in the laboratory system. Products of the reaction on the mylar target $\left(\mathrm{C}_{10} \mathrm{H}_{8} \mathrm{O}_{4}\right)$ with a thickness of $134 \mu \mathrm{g} / \mathrm{cm}^{2}$ were detected by solid state $\mathrm{dE}-\mathrm{E}$ telescopes with thicknesses 150 and 2,000 $\mu \mathrm{m}$.

The second measurement was realized at the Texas A\&M University K500 superconducting cyclotron with a $29.71 \mathrm{MeV}$ ${ }^{3} \mathrm{He}$ beam, with the mylar target (thickness $540 \mu \mathrm{g} / \mathrm{cm}^{2}$ ) at small angles between $1^{\circ}$ and $11^{\circ}$. A Multipole Dipole Multipole magnetic spectrometer (MDM) was used for the detection of reaction products. A gas ionization chamber $(50 \mathrm{~cm}$ long) was placed at the MDM focal plane and was followed by NE102A plastic scintillator, where the residual energy was measured.

Theoretical analysis was performed using the code PTOLEMY with seven different parameter sets for the entrance 


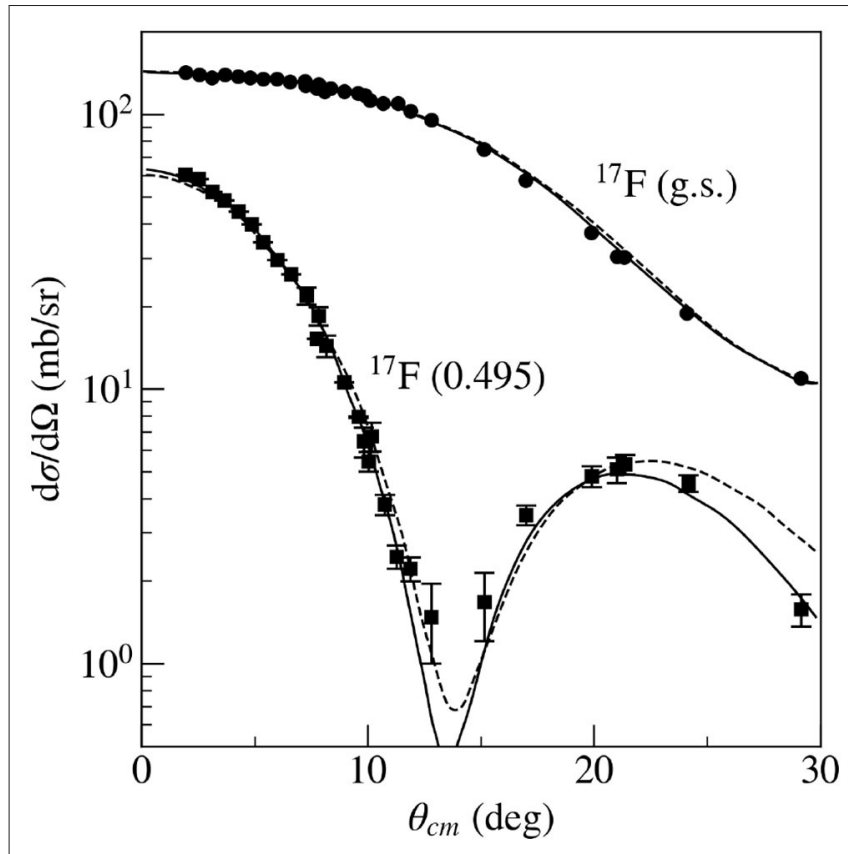

FIGURE 4 | Angular distributions for the ground and first excited states of ${ }^{17} \mathrm{~F}$ from the ${ }^{16} \mathrm{O}\left({ }^{3} \mathrm{He}, \mathrm{d}\right){ }^{17} \mathrm{~F}$ reaction. The dashed and solid curves are DWBA fits using two optical model potential parameter sets (Gagliardi et al., 1999).

channel and five parameter sets for the exit channel. The experimental angular distributions corresponding to the ground and first excited state of ${ }^{17} \mathrm{~F}$ together with the best fits of OMP parameters are given in Figure 4. The conditions of peripherality were verified by changing the radius $r_{\text {cutoff }}$. Angular distributions were changing very slightly within limits from 0 to $3 \mathrm{fm}$ (inner part of ${ }^{16} \mathrm{O}$ ). Similarly, the change of the shape of the potential well of the captured proton did not change the corresponding angular distribution too much and consequently also $C^{2}$ (see Figure 4). The value of $C_{3 \mathrm{He}, d}^{2}=3.90 \pm 0.06 \mathrm{fm}^{-1}$ was used from Mukhamedzhanov et al. (1995), where it was determined by a careful analysis based on hundreds of experimental measurements. Final derived results of ANCs gave the value $C_{d_{5 / 2}}^{2}=1.08 \pm 0.10 \mathrm{fm}^{-1}$ for the ground state, and the value $C_{s_{1 / 2}}^{2}=6,490 \pm 680 \mathrm{fm}^{-1}$ for the first excited state. With these ANC values, the astrophysical S-factors for the ground and first excited state of ${ }^{17} \mathrm{~F}$ were calculated and theoretical curves with the experimental values of S-factors are given in Figure 5. The agreement between experimental values of S-factors and direct measurements are very good for the interval below $1 \mathrm{MeV}$. For higher energies the agreement for the first excited state is worse because of the increasing role of the nuclear interior.

The assumptions of applicability of the ANC method are satisfied for the ${ }^{16} \mathrm{O}(p, \gamma){ }^{17} \mathrm{~F}$ reaction and the method was shown to work in real cases.

\section{3. $\left.{ }^{7} \mathrm{Be}(p, \gamma)\right)^{8} \mathrm{~B},{ }^{13} \mathrm{C}(p, \gamma){ }^{14} \mathrm{~N}$}

The importance of the proton radiative capture $\mathrm{p}+{ }^{7} \mathrm{Be}$ was discussed in the previous section. Two suitable reactions for

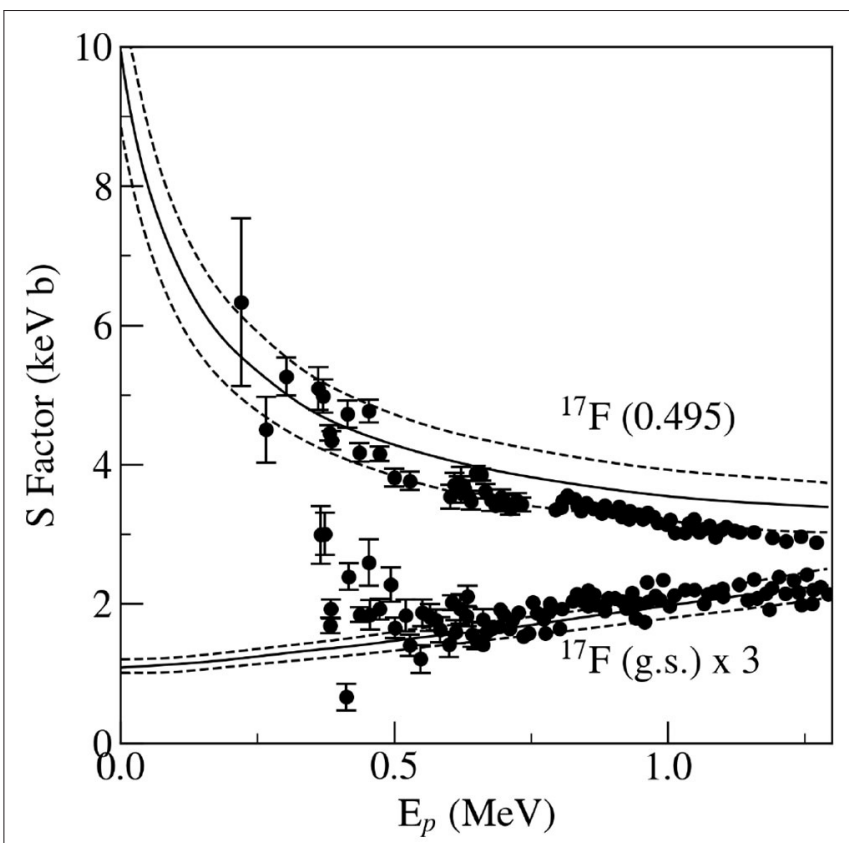

FIGURE 5 | The experimental S-factors and S-factors determined from the ANCs of the ${ }^{16} \mathrm{O}\left({ }^{3} \mathrm{He}, \mathrm{d}\right){ }^{17} \mathrm{~F}$ reaction (Gagliardi et al., 1999). The solid data points are from Morlock et al. (1997), and the open boxes are from Chow et al (1975). The solid lines indicate the calculated S-factors, and the dashed lines correspond to the $\pm 1 \sigma$ error bands.

the indirect determination of ${ }^{7} \mathrm{Be}$ were chosen: the reaction ${ }^{10} \mathrm{~B}\left({ }^{7} \mathrm{Be},{ }^{8} \mathrm{~B}\right){ }^{9} \mathrm{Be}$ which was discussed above and also the transfer reaction ${ }^{14} \mathrm{~N}\left({ }^{7} \mathrm{Be},{ }^{8} \mathrm{~B}\right){ }^{13} \mathrm{C}$. Measurements of angular distributions of elastic scattering of ${ }^{7} \mathrm{Be}$ ions and also, of transfer reactions with these ions on the ${ }^{10} \mathrm{~B}$ and ${ }^{14} \mathrm{~N}$ targets were carried out on the K500 superconducting cyclotron at Texas A\&M University (Azhari et al., 1999, 2001).

The beam of radioactive ${ }^{7}$ Be ions was produced in the ${ }^{1} \mathrm{H}\left({ }^{7} \mathrm{Li}\right.$, $\left.{ }^{7} \mathrm{Be}\right) \mathrm{n}$ reaction using ${ }^{7} \mathrm{Li}$ primary beam of $135 \mathrm{MeV}$ energy. The ${ }^{7} \mathrm{Li}$ beam was striking on the liquid nitrogen cooled gas cell containing hydrogen with pressure about $1 \mathrm{~atm}$. Windows of the gas cell were made from Havar foil with a $42 \mathrm{mg} / \mathrm{cm}^{2}$ thickness. ${ }^{7} \mathrm{Be}$ ions from the gas cell were separated by the Momentum Achromat Recoil Spectrometer (MARS) and hit the target with a rate below $10^{5}$ pps. The self-supported ${ }^{10} \mathrm{~B}$ target was prepared from a mixture of pulverized ${ }^{10} \mathrm{~B}$ and varnish deposited on a Ta backing from which the target layer was removed in distilled water. As the ${ }^{14} \mathrm{~N}$ target the evaporated melamine $\left(\mathrm{C}_{3} \mathrm{~N}_{6} \mathrm{H}_{6}\right)$ on a $20 \mu \mathrm{g} / \mathrm{cm}^{2}$ layer of $\mathrm{C}$ and a $20 \mu \mathrm{g} / \mathrm{cm}^{2}$ layer of collodion was used. The average thicknesses of the targets were $1.96 \mathrm{mg} / \mathrm{cm}^{2}$ and $1.50 \mathrm{mg} / \mathrm{cm}^{2}$ for the ${ }^{10} \mathrm{~B}$ and ${ }^{14} \mathrm{~N}$ targets. Reaction products were measured and identified by the telescope configuration consisting of a $100 \mu \mathrm{m}$ silicon strip detector, backed by a $1,000 \mu \mathrm{m} 5 \times 5 \mathrm{~cm}^{2} \mathrm{Si}$ detector. Data from the telescope were then analyzed to deduce angular distributions of the elastic scattering of ${ }^{7} \mathrm{Be}$ and the studied transfer reactions. The angular distribution of elastic scattering was used for determination of optical model parameters. The parameters were obtained from 


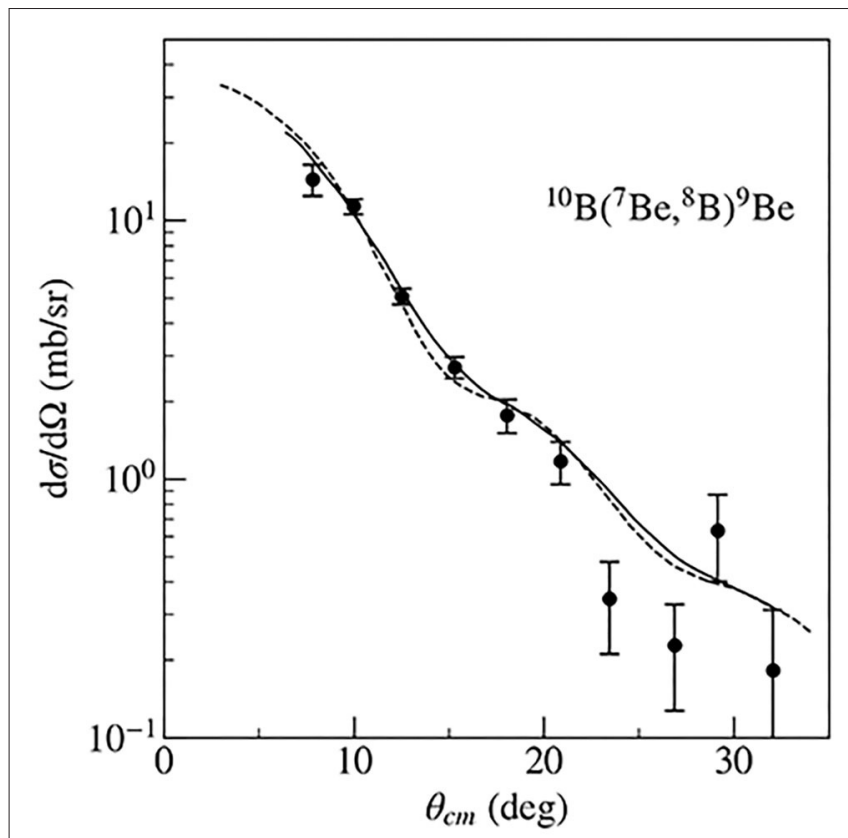

FIGURE 6 | Experimental angular distribution from the ${ }^{10} \mathrm{~B}\left({ }^{7} \mathrm{Be},{ }^{8} \mathrm{~B}\right){ }^{9} \mathrm{Be}$ reaction for the outgoing ions ${ }^{7} \mathrm{Be}$. The dashed line corresponds to the predicted angular distribution and the solid line is corrected to finite angular distribution (Azhari et al., 1999).

double folding model calculations according to JLM effective interaction (Jeukenne et al., 1977).

Having the optical model parameters for the input channel and exit channels ${ }^{10} \mathrm{~B}+{ }^{7} \mathrm{Be}$ and ${ }^{8} \mathrm{~B}+{ }^{9} \mathrm{Be}$, it was possible to calculate the differential cross-section for the transfer reaction. Similarly for the transfer reaction ${ }^{14} \mathrm{~N}\left({ }^{7} \mathrm{Be},{ }^{8} \mathrm{~B}\right){ }^{13} \mathrm{C}$, where model parameters for the input channel ${ }^{7} \mathrm{Be}+{ }^{14} \mathrm{~N}$ and the exit channel ${ }^{8} \mathrm{~B}+{ }^{13} \mathrm{C}$ were needed. The experimental angular distribution for the outgoing ${ }^{8} \mathrm{~B}$ of the ${ }^{10} \mathrm{~B}\left({ }^{7} \mathrm{Be},{ }^{8} \mathrm{~B}\right){ }^{9} \mathrm{Be}$ reaction $(\mathrm{Q} \leq 28 \mathrm{MeV})$ is shown in Figure 6. The calculations of theoretical angular distributions were performed by the code PTOLEMY. The peripheral character of both transfer reactions ${ }^{10} \mathrm{~B}\left({ }^{7} \mathrm{Be},{ }^{8} \mathrm{~B}\right){ }^{9} \mathrm{Be}$ and ${ }^{14} \mathrm{~N}\left({ }^{7} \mathrm{Be},{ }^{8} \mathrm{~B}\right){ }^{13} \mathrm{C}$ was tested using variations of radius and diffuseness values of the single-particle Woods-Saxon potential. The changes in deduced ANCs were only $\pm 3.5 \%$. The deduced value $C_{13}^{2} C+p$, g.s. $=19.5 \pm 2.5 \mathrm{fm}^{-1}$, values for other states are shown in Trache et al. (1998), Table 2. The ANC value for ${ }^{7} \mathrm{Be}+\mathrm{p}$ reaction is $C_{7}^{2} \mathrm{Be}+p=0.388 \pm 0.039 \mathrm{fm}^{-1}$. Using the ANCs from both transfer reactions ${ }^{10} \mathrm{~B}\left({ }^{7} \mathrm{Be},{ }^{8} \mathrm{~B}\right){ }^{9} \mathrm{Be}$ and ${ }^{14} \mathrm{~N}\left({ }^{7} \mathrm{Be},{ }^{8} \mathrm{~B}\right){ }^{13} \mathrm{C}$, astrophysical S-factors $S_{17}(0)=18.4 \pm 2.5 \mathrm{eV}$ b and $S_{17}(0)=16.9$ $\pm 1.9 \mathrm{eV}$ b was obtained. The weighted average value $S_{17}(0)=$ $17.3 \pm 1.8 \mathrm{eV}$ b is included in the Figure 7 (Azhari et al., 2001). This is in good agreement with the current accepted value $S_{17}(0)$ $=19_{-2}^{+4} \mathrm{eV}$ b.

Solid targets, such as ${ }^{14} \mathrm{~N}$ in the previous section, are available in the form of compounds and/or with other element backings. This may substantially complicate an analysis, due to peak overlap in spectra and different kinematical shifts for isotopes with different mass numbers. One of the experimental challenges was ANC measurement with pure isotopic gas targets.

The assumptions of applicability of the ANC method are satisfied for the ${ }^{7} \mathrm{Be}(p, \gamma){ }^{8} \mathrm{~B}$ reaction. The ANC of ${ }^{13} \mathrm{C}(p, \gamma){ }^{14} \mathrm{~N}$ reaction, due to the applicability conditions, was only used for the analysis of ${ }^{7} \mathrm{Be}(\mathrm{p}, \gamma)^{8} \mathrm{~B}$ and other captures (see below).

\section{4. ${ }^{20} \mathrm{Ne}(\boldsymbol{p}, \gamma)^{21} \mathrm{Na}$}

The ${ }^{20} \mathrm{Ne}(p, \gamma){ }^{21} \mathrm{Na}$ capture reaction is a part of the so-called $\mathrm{NeNa}$-cycle, which takes place in stars larger than the Sun. This cycle produces ${ }^{21} \mathrm{Na},{ }^{21} \mathrm{Ne},{ }^{22} \mathrm{Na}$, and ${ }^{22} \mathrm{Ne}$ nuclei, while consuming hydrogen. The ${ }^{20} \mathrm{Ne}\left({ }^{3} \mathrm{He}, d\right){ }^{21} \mathrm{Na}$ reaction leads to four bound states of ${ }^{21} \mathrm{Na}$. The highest lying state with excitation energy of $2.425 \mathrm{MeV}$ is only a few $\mathrm{keV}$ below threshold The capture to this subthreshold state dominates the value of astrophysical S-factor.

The measurement was realized on the beam of the isochronous cyclotron U120M of NPI CAS. The ${ }^{3} \mathrm{He}$ beam with energy $25.83 \mathrm{MeV}$ impinged the target gas cell filled with high purity ${ }^{20} \mathrm{Ne}$. The input and output windows were made of $3.05 \mu \mathrm{m}$ Havar foils. The working pressure was kept at 195 mbar and was continuously monitored together with the gas temperature. The detection system consisted of a pair of $\mathrm{dE}-\mathrm{E}$ $\mathrm{Si}(\mathrm{Li})$ surface barrier detectors of thicknesses $220 \mu \mathrm{m}$ and $4 \mathrm{~mm}$, respectively. The effective thickness of the gas target seen by the telescopes was obtained from a simulation. One telescope at a fixed angle served as a monitor and the second telescope was movable in the angular interval from $6.5^{\circ}$ to $70^{\circ}$. The obtained energy resolution ranged from 100 to $120 \mathrm{keV}$, depending on the measured angle.

The experimental angular distributions of emitted deuterons from the ${ }^{20} \mathrm{Ne}\left({ }^{3} \mathrm{He}, \mathrm{d}\right){ }^{21} \mathrm{Na}$ reaction were analyzed within the DWBA theory. At first the angular distribution of elastic scattering of ${ }^{3} \mathrm{He}$ was fitted by means of the code ECIS79 (Raynal, 1981) to obtain optical model parameters for the input channel. The optical model parameters for the exit channel were adopted from global formulas in works by Daehnick et al. (1980) and Vernotte et al. (1994). In Figure 8 we present, as an example, the experimental angular distribution fits for the state $2.425 \mathrm{MeV}$ calculated by the code DWUCK5. The peripheral character of the reaction was verified using different cut-off radii of the integration over the radial part of interaction. The stability check of the function $R(b)$ (9) was checked and its behavior was almost flat. To deduce ANCs for the direct capture ${ }^{20} \mathrm{Ne}(\mathrm{p}, \gamma){ }^{21} \mathrm{Na}$ from the transfer reaction ${ }^{20} \mathrm{Ne}\left({ }^{3} \mathrm{He}, \mathrm{d}\right){ }^{21} \mathrm{Na}$, knowledge of ANC for the decay ${ }^{3} \mathrm{He} \rightarrow \mathrm{d}+\mathrm{p}$ is necessary. For further analysis we used the value $\left(C_{d p}^{3} \mathrm{He}\right)^{2}=3.90 \pm 0.06 \mathrm{fm}^{-1}$ (Mukhamedzhanov et al., 1995).

Total uncertainties of ANC for the direct capture ${ }^{20} \mathrm{Ne}(\mathrm{p}, \gamma){ }^{21} \mathrm{Na}$ were estimated from $14 \%$ for the $2.425 \mathrm{MeV}$ state to $28 \%$ for the $1.716 \mathrm{MeV}$ state. The sources were mainly (1) the ambiguity of optical model parameters and (2) the uncertainty of absolute values of the cross-sections. R-matrix theory was used to determine S-factor, where the normalization of the direct capture amplitude was given by the ANC of the final bound state according the procedure used by Tang et al. (2003). The 
TABLE 2 | Squared ANC values (in units of $\left[\mathrm{fm}^{-1}\right]$ ) for selected reactions discussed in this paper (column $C_{1}^{2}$ ).

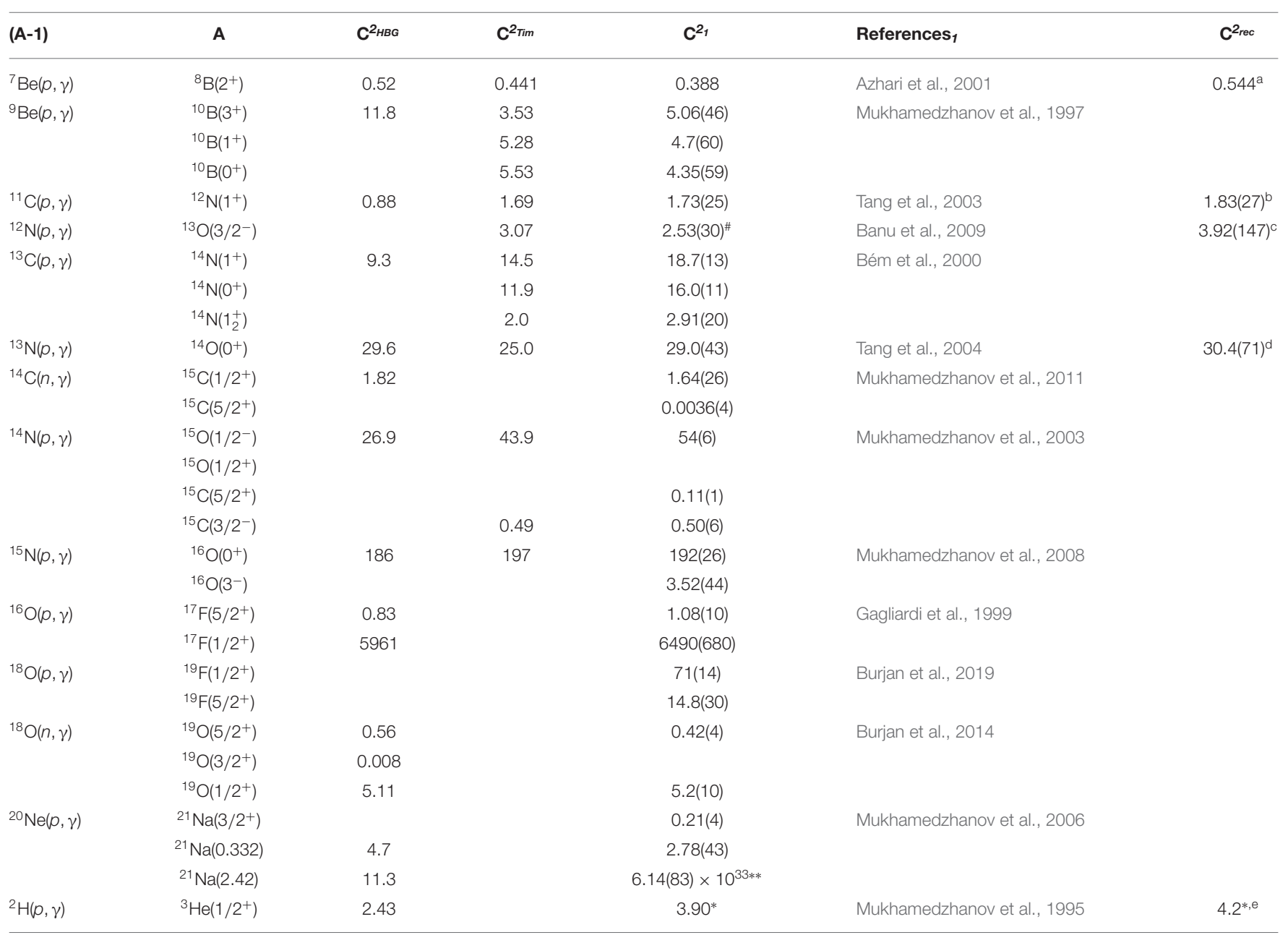

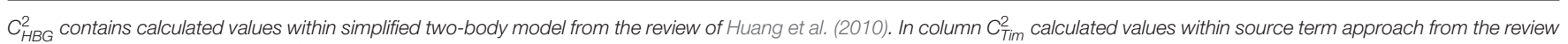
of Timofeyuk (2013) are shown. $C_{\text {rec }}^{2}$ column contains more recent experimental values, where available.

a From Trache et al. (2003).

${ }^{b}$ From Lee et al. (2011).

${ }^{c}$ From Guo et al. (2013).

${ }^{d}$ From Bing and Zhi-Hong (2007), determined from the mirror reaction.

eFrom Yarmukhamedov and Blokhintsev (2018).

\# Only $C_{p 1 / 2}^{2}$ was published in Banu et al. (2009) and it agrees with $C_{p 1 / 2, T i m}^{2}=2.60$ of.

* Determined theoretically (values were/are used in deduction of the other ANCs).

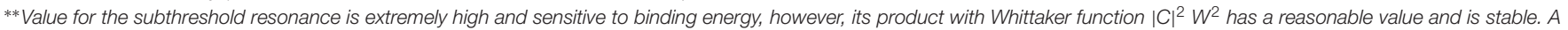
reason for the discrepancy with the model is uncertain (Huang et al., 2010).

$S$-factor for direct capture to the subthreshold state was found to be $S(0)=68.30 \pm 9.30 \mathrm{keV}$ b. The direct capture to the ground state is very weak. The capture to the ground state is dominated by the resonant capture through the subthreshold state. It was determined as $\mathrm{S}(0)=5,870.0 \pm 1,200 \mathrm{keV}$ b. The $\mathrm{R}$-matrix calculations were performed with the channel radius $r=5 \mathrm{fm}$. The calculated total $\mathrm{S}(\mathrm{E})$ consists of the sum of the direct capture to the subthreshold state and the capture by the subthreshold resonance to the ground state. We have thus $S(0)$ $=5,900 \pm 1,200 \mathrm{keV} b$ (Figure 9). Contributions to the total $\mathrm{S}$-factor from captures to other remaining states are negligible. The newly obtained $S(0)$ factor is higher than the value given in Rolfs and Rodney (1988).
The proton partial width of the resonance, dominating the radiative capture of the ${ }^{20} \mathrm{Ne}(p, \gamma)^{21} \mathrm{Na}$ reaction was deduced from the measured ANC.

\section{5. ${ }^{18} \mathrm{O}(n, \gamma)^{19} \mathrm{O}$}

The ANC method can be used not only for charged particle direct captures, but also, for a direct radiative neutron captures (Imai et al., 2001). However, the peripherality of the process must be ensured by a presence of a centrifugal barrier. The ${ }^{18} \mathrm{O}(\mathrm{n}, \gamma){ }^{19} \mathrm{O}$ capture is given as an example.

The inhomogeneous Big Bang models (IBBN) seemed to contain a solution for the problems of BBN. Nuclei with A $>12$, in neutron-rich environments, could be formed in 


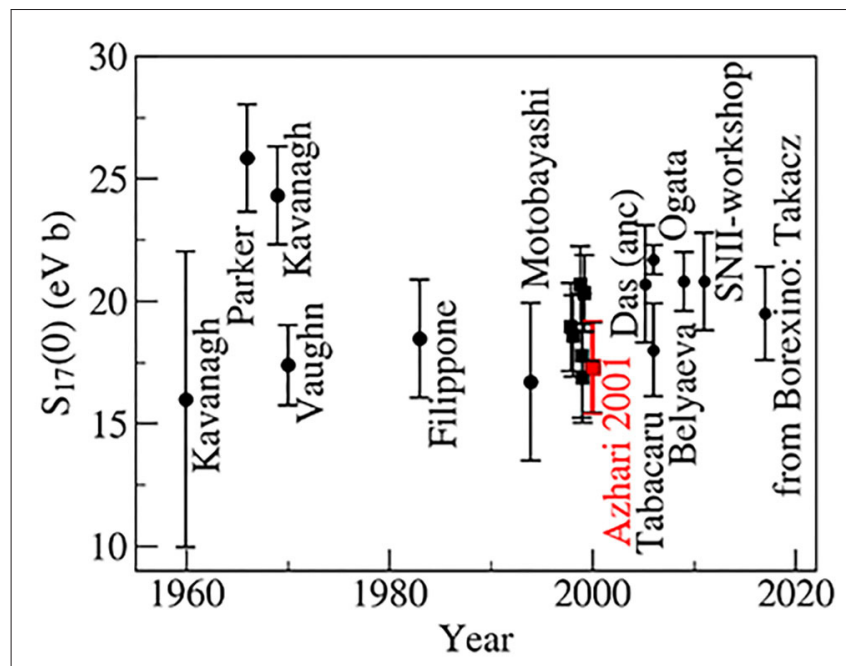

FIGURE 7 | History of the determination of astrophysical $S_{17}(0)$. The value measured in the experiment, discussed in the text, is in red and labeled "Azhari 2001." For details see (Azhari et al., 2001).

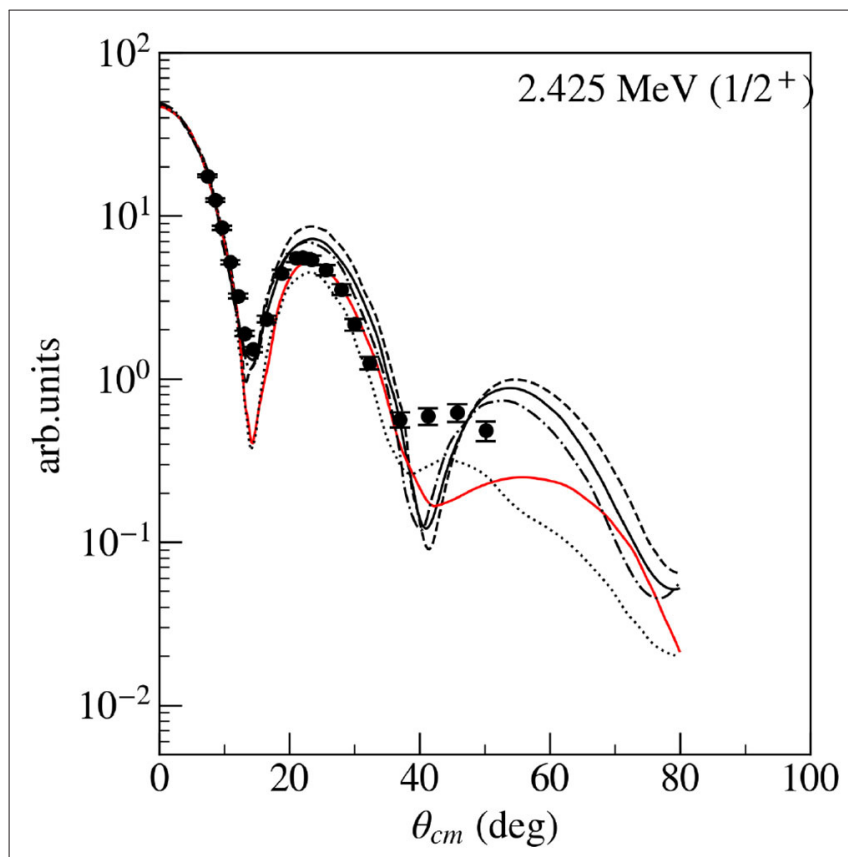

FIGURE 8 | The angular distribution of the ${ }^{20} \mathrm{Ne}\left({ }^{3} \mathrm{He}, \mathrm{d}\right)^{21} \mathrm{Na}$ reaction corresponding to the transition to the subthreshold state of ${ }^{21} \mathrm{Na}$ fitted by combinations of different optical model parameters for input and output channels. OM parameters for input and output channels taken from earlier works produce the black solid curve. The black dashed and dash-dotted curves use the same output channel OM parameters, but the input channel $\mathrm{OM}$ parameters were obtained from a fit of the elastic scattering data with two different seeds. Black dotted and red curves are produced as the two previous curves, but with a different OM parameter set for the output channel. For details see (Mukhamedzhanov et al., 2006).

the reaction sequence (Wiescher et al., 1990) ${ }^{14} \mathrm{C}(n, \gamma){ }^{15} \mathrm{C}\left(\beta^{-}\right)$ ${ }^{15} \mathrm{~N}(n, \gamma){ }^{16} \mathrm{~N}\left(\beta^{-}\right){ }^{16} \mathrm{O}(n, \gamma){ }^{17} \mathrm{O}(n, \gamma){ }^{18} \mathrm{O}(n, \gamma){ }^{19} \mathrm{O}$, where the reaction ${ }^{18} \mathrm{O}(\mathrm{n}, \gamma){ }^{19} \mathrm{O}$ opens the path to elements with higher

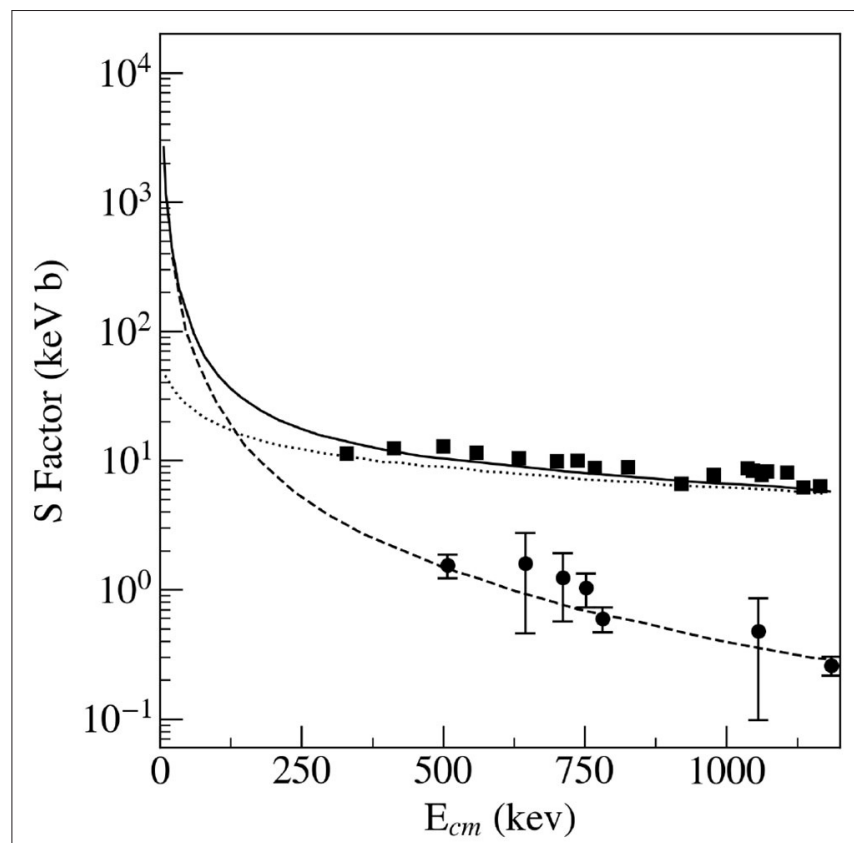

FIGURE 9 | The astrophysical S-factor for the direct ${ }^{20} \mathrm{Ne}(\mathrm{p}, \gamma)^{21} \mathrm{Na}$ capture. The solid squares and dotted curve are experimental data points from Rolfs et al. (1975) and results of Mukhamedzhanov et al. (2006) for direct capture to the subthreshold state, respectively. Similarly, for the capture to the ground state the open squares and dashed curve correspond to the experimental data points and to results for the direct capture. The determined total astrophysical S-factor is given by the solid curve.

mass numbers (Wiescher et al., 1990). The neutron radiative capture ${ }^{18} \mathrm{O}(\mathrm{n}, \gamma){ }^{19} \mathrm{O}$ also has a significance for the estimation of reaction rates of stellar helium burning in massive red stars and AGB stars. To determine the direct $(n, \gamma)$ cross-section, a precise measurement of $(\mathrm{d}, \mathrm{p})$ differential cross-section on ${ }^{18} \mathrm{O}$ target at forward angles (where the stripping mechanism is more pronounced) was required. Although, the transfer to higher excited states was also of interest (Herndl et al., 1999), not all were possible to resolve. The experiment was performed on the isochronous cyclotron U-120M of the NPI CAS (Burjan et al., 2013). A momentum analyzed 16.3 MeV deuteron beam impinged on an oxygen gas target. The gas chamber target was filled with a high purity ${ }^{18} \mathrm{O}$ isotope (99.9\%). The working pressure was maintained at 150 mbar. Eight dEE telescopes [250 $\mu \mathrm{m}$ and $5 \mathrm{~mm}$ thick $\mathrm{Si}(\mathrm{Li})$ surface barrier detectors] were used to register the reaction products. DWBA analysis of experimental angular distributions of the transfer reaction ${ }^{18} \mathrm{O}(\mathrm{d}, \mathrm{p}){ }^{19} \mathrm{O}$ was performed to extract ANCs. The phenomenological optical potential of Kunz (1990) has been used for the analysis of the angular distributions. Input channel parameters were obtained by the fit of the experimental angular distribution of elastically scattered deuterons. For this purpose, the code ECIS79 of Raynal (1979) was used. Optical model parameter sets of Perey and Perey (1976) and others (Duke, 1963; Watson et al., 1969) were used for the proton exit channel. The experimental angular distributions ${ }^{18} \mathrm{O}(\mathrm{d}, \mathrm{p}){ }^{19} \mathrm{O}$ 


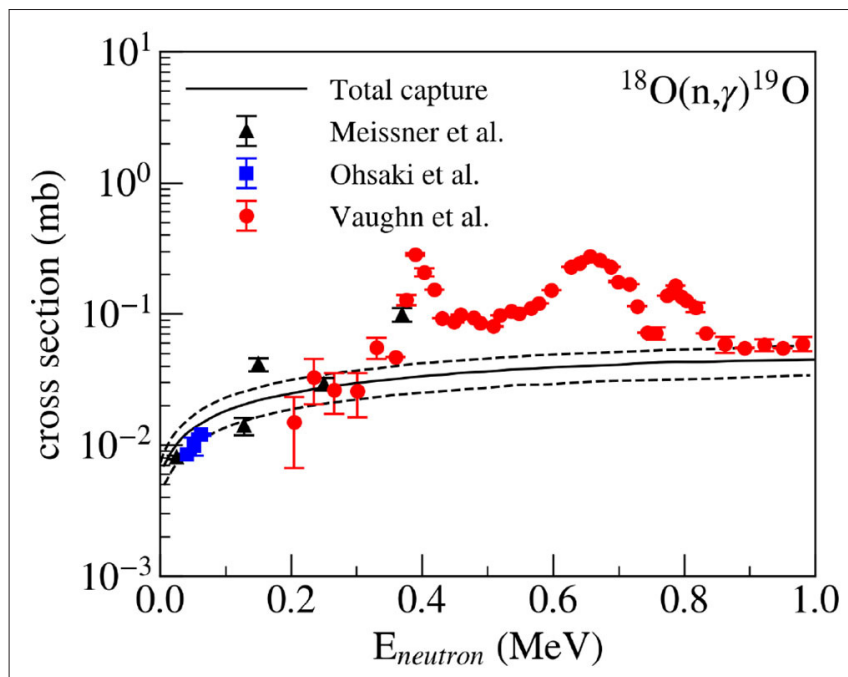

FIGURE 10 | Comparison of the cross-section of the direct radiative neutron capture ${ }^{18} \mathrm{O}(\mathrm{n}, \gamma){ }^{19} \mathrm{O}$ determined by the ANC method from the ${ }^{18} \mathrm{O}(\mathrm{d}, \mathrm{p})^{19} \mathrm{O}$ reaction with experimental data of Vaughn et al. (1965), Meissner et al. (1996), and Ohsaki et al. (2008). Dashed lines show the uncertainty band 22\%.

were fitted within the DWBA theory using the DWUCK5 code (by Kunz, 1990). As in the above cases, two overlap integrals and two ANCs are contained in the DWBA description of this reaction. The ANC value for the virtual decay $d \rightarrow p+n$ is $C_{p n}^{2}=0.77 \mathrm{fm}^{-1}$, based on considerations about spectroscopic factor $S=1$ and SPANC values at an asymptotic distance (Mukhamedzhanov et al., 2011).

For transferred neutral particle, instead of the Whittaker function, the Hankel function is used in the overlap integral in the asymptotic region. For the application of the ANC method, the condition of the weak cross-section dependence on the cut-off radius should be fulfilled. The difference of the crosssection at the maximum of the angular distribution was $13 \%$ between the case with $3 \mathrm{fm}$ cut-off and no cut-off for the transfer to the ground state. Changes were negligible (2\%) for other transitions. The obtained values $C_{A n l j}^{2}$ for the transitions to five states of ${ }^{19} \mathrm{O}$ (g. s., 1.471, 3.153, 3.231, and $3.944 \mathrm{MeV}$ ) were used to estimate the direct neutron capture contribution by ${ }^{18} \mathrm{O}$ (see in Burjan et al., 2013). The FRESCO code (Thompson, 1988) was used for these $(\mathrm{n}, \gamma)$ calculations. In the calculations, the channel radius $3.3 \mathrm{fm}$ was used and the $E 1$ multipolarity was assumed in all cases. The largest contribution (black solid line in Figure 10) is from transition to the $1.471 \mathrm{MeV}$ state $\left(2 s_{1 / 2}\right)$. The results were compared with available experimental data (Figure 10). Data points from Meissner et al. (1996) and Ohsaki et al. (2008) for the transitions to the ground state and $1.471 \mathrm{MeV}$ state are shown. The larger energy interval was measured by Vaughn et al. (1965) (transitions to the g. s. and $1.471 \mathrm{MeV}$ state). The direct part of the experimental data is quite consistent with the ANC method results for this neutron capture by ${ }^{18} \mathrm{O}$.

The necessity of the centrifugal barrier allowed studying the p-wave direct capture contribution with the ANC method.
Direct measurements show that it dominates in the energies bellow $100 \mathrm{keV}$.

\section{6. ${ }^{18} \mathrm{O}(p, \gamma){ }^{19} \mathrm{~F}$}

A study of the radiative capture reaction ${ }^{18} \mathrm{O}(\mathrm{p}, \gamma){ }^{19} \mathrm{~F}$ helps in better understanding the processes in AGB stars (i. e. stars several times heavier than our Sun). The hydrogen burning of ${ }^{18} \mathrm{O}$ via the $(\mathrm{p}, \gamma)$ in these stars is competing with the ${ }^{18} \mathrm{O}(\mathrm{p}, \alpha)^{19} \mathrm{~F}$ reaction (Lorenz-Wirzba et al., 1979). The (p, $\gamma$ ) capture causes the escape of ${ }^{19} \mathrm{~F}$ from $\mathrm{CNO}$ cycles, while the $(\mathrm{p}, \alpha)$ reaction (with a rate of about 3 orders higher at solar energies) returns the ${ }^{19} \mathrm{~F}$ back. The ratio ${ }^{18} \mathrm{O} /{ }^{16} \mathrm{O}$ represents an important parameter that is characteristic for different nucleosynthesis locations and that can help to determine the origin of e.g., presolar grains (Abia et al., 2017; Palmerini et al., 2017). While the direct part of the ${ }^{18} \mathrm{O}$ (p, $\gamma)$ process is not the major contribution to radiative capture, it may play a role in the total S-factor, via interferences with resonant states. A measurement of the ${ }^{18} \mathrm{O}(\mathrm{p}, \gamma)^{19} \mathrm{~F}$ in the energy range $E_{p}=0.08-2.2 \mathrm{MeV}$ was performed by Wiescher et al. (1980), where the direct part of the (p, $\gamma)$ capture was determined experimentally and also calculated theoretically. Later, Buckner et al. (2012) investigated reaction rates of ${ }^{18} \mathrm{O}(\mathrm{p}, \gamma){ }^{19} \mathrm{~F}$ and determined the direct part of this capture using different capture models. However, there is a substantial difference between the astrophysical S-factor of Wiescher et al. and of Buckner et al. at low energies. The energy dependence of values of Wiescher et al. decreases while that of Buckner et al. is increases. Also, their absolute values differ considerably. We therefore, decided to determine the astrophysical S-factor of the direct capture ${ }^{18} \mathrm{O}(\mathrm{p}, \gamma){ }^{19} \mathrm{~F}$ by the ANC method. The differential cross-section of the ${ }^{18} \mathrm{O}\left({ }^{3} \mathrm{He}, \mathrm{d}\right){ }^{19} \mathrm{~F}$ transfer reaction was measured (Burjan et al., 2019). 24.6 $\mathrm{MeV}^{3} \mathrm{He}$ beam from the isochronous cyclotron $\mathrm{U}-120 \mathrm{M}$ at NPI CAS was used. High purity ${ }^{18} \mathrm{O}$ oxygen (99.9\%) gas target was used as in the above experiment. Eight dEE telescopes $[250 \mu \mathrm{m}$ and $5 \mathrm{~mm}$ thick $\mathrm{Si}(\mathrm{Li})$ surface barrier detectors] were used for detection of the reaction products. The geometry of detectors was very similar to the geometry used in the $(\mathrm{d}, \mathrm{p})$ experiment. Twelve deuteron peaks corresponding to the bound states of the ${ }^{19} \mathrm{~F}$ were observed and analyzed. Other ${ }^{19} \mathrm{~F}$ levels were populated weakly and were not considered further. The phenomenological optical potential was used for the analysis of the measured angular distributions. The input channel OM parameter sets were deduced from the fit of the angular distribution of elastic scattering ${ }^{3} \mathrm{He}+{ }^{18} \mathrm{O}$. The output channel OM parameters for deuterons were adopted from the global formula of Perey and Perey (1976). The FRESCO code (Thompson, 1988) was used to calculate the theoretical angular distributions of the ${ }^{18} \mathrm{O}\left({ }^{3} \mathrm{He}, \mathrm{d}\right){ }^{19} \mathrm{~F}$ transfer reaction. The transitions with the largest ANCs are to the ground state and to the 0.197 and $1.554 \mathrm{MeV}$ levels of ${ }^{19} \mathrm{~F}, C_{18}^{2} \mathrm{O}+$ p, g.s. $=71.1 \pm$ $14 \mathrm{fm}^{-1}, C_{18}^{2} \mathrm{O}+p, 0.197=14.8 \pm 3 \mathrm{fm}^{-1}$. The derived ANCs were used to deduce the direct capture cross-section $\sigma(E)_{\text {cap }}$ by the FRESCO code The dependence of the differential cross-section on the cut-off radius was tested to verify the surface character of the transfer reaction. E1 multipole electromagnetic operator was considered in the calculations. Woods-Saxon potential was used 


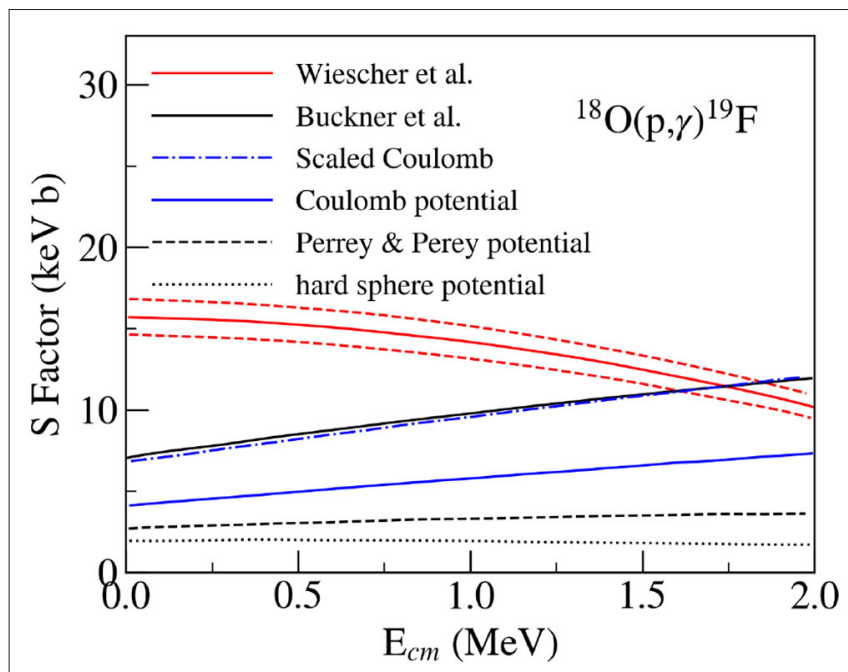

FIGURE 11 | S-factor of the total direct proton capture ${ }^{18} \mathrm{O}(\mathrm{p}, \gamma){ }^{19} \mathrm{~F}$ determined from the transfer reaction. The red solid decreasing curve is based on the measurement of the direct contribution ( $p, \gamma)$ by Wiescher et al. (1980) with uncertainty about $7 \%$ (dashed red curves).

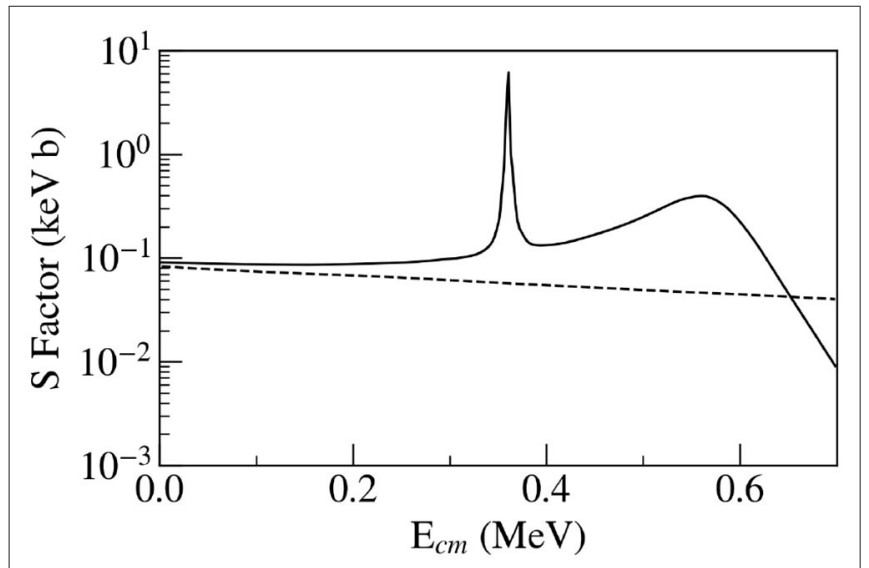

FIGURE 12 | The astrophysical S-factor for ${ }^{11} \mathrm{C}(\mathrm{p}, \gamma){ }^{12} \mathrm{~N}$, the total S-factor (solid line) and the direct contribution alone (dotted line).

for calculation of the bound state wave functions (Wiescher et al., 1980). Three kinds of potentials for scattered wave functions of incoming protons were: (1) the complex optical potential of Perey and Perey (1976), (2) the Coulomb potential only (which plays a major role at low proton energies) and (3) the hard sphere potential $(V=-300 \mathrm{MeV})$ simulating a repulsive potential. The total direct $\mathrm{S}$-factors for all three potentials are shown in Figure 11. The capture to the ground and $0.197 \mathrm{MeV}$ states dominates the total direct S-factor (60\% contribution). These S-factors were compared with calculations of ${ }^{18} \mathrm{O}(\mathrm{p}, \gamma){ }^{19} \mathrm{~F}$ by Buckner et al. (2012) and with the direct measurement by Wiescher et al. (1980) at the low-energy region. It appears that the direct hard sphere potential $\mathrm{S}(\mathrm{E})$-factor is almost constant ( $2 \mathrm{keV} \mathrm{b}$ ), while the others have the tendency to rise slowly in the interval between 0 and $2 \mathrm{MeV}$, in agreement with Buckner et al.
$\left.((S) E)=7.06+2.98 \times 10^{-3} E-2.6 \times 10^{-7} E^{2}[\mathrm{keV} \mathrm{b}]\right)$ and in disagreement with Wiescher et al. $((S) E)=15.7-0.34 \times 10^{-3} E-$ $1.21 \times 10^{-6} E^{2}[\mathrm{keV}$ b]) The calculation of the direct S-factor with the Coulomb potential when normalized to the measured direct capture cross-section at $E_{c . m} .=1751.9 \mathrm{keV}$ by Wiescher et al. (1980) is in a good agreement with the result of Buckner et al. The total direct astrophysical S-factor calculated with three different interaction potentials show a tendency similar to that of Buckner et al. For the Coulomb interaction potential, the calculations reproduce these data very well, after normalization. Without normalization, they are lower than the values of Buckner et al. at least about 35\%.

Due to the large number of resonances, the applicability conditions are not satisfied. The ANC value of ${ }^{18} \mathrm{O}(p, \gamma){ }^{19} \mathrm{~F}$ reaction can be compared with the different calculations used for determination of the direct $\mathrm{S}$-factor.

\section{7. ${ }^{11} \mathrm{C}(p, \gamma){ }^{12} \mathrm{~N}$}

The reaction ${ }^{14} \mathrm{~N}\left({ }^{11} \mathrm{C},{ }^{12} \mathrm{~N}\right){ }^{13} \mathrm{C}$ can provide ANC for the ${ }^{12} \mathrm{~N} \rightarrow$ ${ }^{11} \mathrm{C}+\gamma$ decay and the direct capture rate for ${ }^{11} \mathrm{C}(\mathrm{p}, \gamma){ }^{12} \mathrm{~N}$ at astrophysical energies. This reaction is a part of the hot pp-chain $\left({ }^{7} \operatorname{Be}(\alpha, \gamma){ }^{11} \mathrm{C}\right)$. It may open a possibility for supermassive, low-metallicity stars to produce CNO nuclei (avoiding the three-alpha process) (Wiescher et al., 1989). The ${ }^{11} \mathrm{C}$ radioactive beam, at an intensity of $4.2 \times 10^{5}$ particles/s and energy $10 \mathrm{MeV} / \mathrm{u}$, bombarded a $1.5 \mathrm{mg} / \mathrm{cm}^{2}$ melamine target $\left(\mathrm{C}_{3} \mathrm{~N}_{6} \mathrm{H}_{6}\right)$ (Gagliardi et al., 2002). This radioactive beam was produced in the reaction ${ }^{1} \mathrm{H}\left({ }^{11} \mathrm{~B},{ }^{11} \mathrm{C}\right) \mathrm{n}$ and purified by the Momentum Achromat Recoil Spectrometer (MARS). S-factor for direct ${ }^{11} \mathrm{C}(\mathrm{p}, \gamma){ }^{12} \mathrm{~N}$ was deduced (Gagliardi et al., 2001) using the R-matrix calculation that includes effects of two low-lying resonances and the determined ANC factor $\left(C_{11}^{2} \mathrm{C}+p=1.73 \pm\right.$ $0.25 \mathrm{fm}^{-1}$ ) (Tang et al., 2003). Results are shown in Figure 12. The values of S-factor are more than 10 times larger than previously given (Lefebvre et al., 1995).

Non-resonant capture is thought to dominate the ${ }^{11} \mathrm{C}(p, \gamma){ }^{12} \mathrm{~N}$ reaction at low energies (Lefebvre et al., 1995; Huang et al., 2010), ANC is an important component for the R-matrix analysis.

\section{8. ${ }^{12} \mathrm{~N}(p, \gamma)^{13} \mathrm{O}$}

The new experimental information on the ${ }^{12} \mathrm{~N}(\mathrm{p}, \gamma){ }^{13} \mathrm{O}$ capture is important for Population II stars with lower masses, modeling a nucleosynthesis and their evolution. This capture reaction was studied by the ANCs indirect method using the peripheral proton-transfer reaction ${ }^{14} \mathrm{~N}\left({ }^{12} \mathrm{~N},{ }^{13} \mathrm{O}\right){ }^{13} \mathrm{C}$ (Banu et al., 2009). The radioactive beam ${ }^{12} \mathrm{~N}$ was produced from a primary beam of ${ }^{12} \mathrm{C}$ at $23 \mathrm{~A} \mathrm{MeV}$ with an intensity of $150 \mathrm{pnA}$ (particle nA) striking on a $\mathrm{LN} 2$-cooled $\mathrm{H}_{2}$ gas cell. To reduce the ${ }^{12} \mathrm{C}$ energy to $12 \mathrm{~A} \mathrm{MeV}$ where the reaction is peripheral a $250-\mu \mathrm{m}$-thick $\mathrm{Al}$ foil was put behind the gas cell. For the DWBA analysis of the transfer reaction ${ }^{14} \mathrm{~N}\left({ }^{12} \mathrm{~N},{ }^{13} \mathrm{O}\right){ }^{13} \mathrm{C}$, the parameters of the optical model potential for both the entrance channel $\left({ }^{12} \mathrm{~N}-{ }^{14} \mathrm{~N}\right)$ and exit channel $\left({ }^{13} \mathrm{O}-{ }^{13} \mathrm{C}\right)$ were needed to calculate the distorted scattering wave functions. Analysis of the elastic scattering data was done with the help of semi-microscopic double-folding optical potentials. The calculated angular distribution of the 


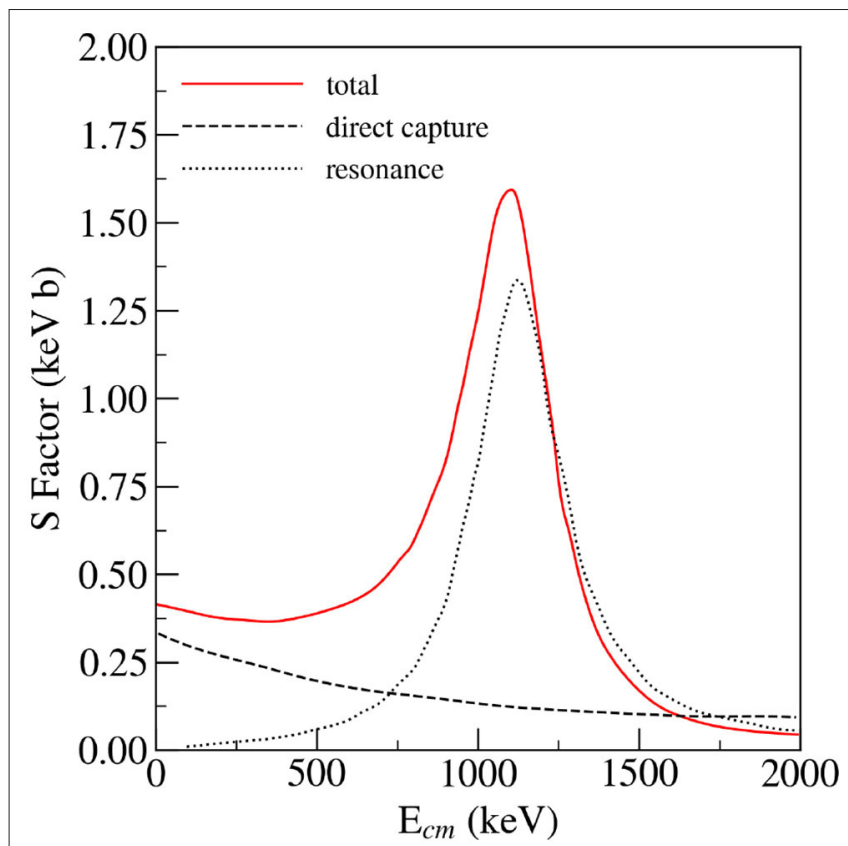

FIGURE 13 | The astrophysical S-factor for the ${ }^{12} \mathrm{~N}(\mathrm{p}, \gamma)^{13} \mathrm{O}$ capture as a function of the energy. The solid red line shows results for the total S-factor, dashed line is the direct component of the S-factor and the dotted line is the resonant component.

transfer reaction ${ }^{14} \mathrm{~N}\left({ }^{12} \mathrm{~N},{ }^{13} \mathrm{O}\right){ }^{13} \mathrm{C}$ by the finite-range DWBA code PTOLEMY was used for the fit of the experimental angular distribution. From the comparison of theoretical and experimental angular distributions, the ANC was determined to be $C_{p_{1 / 2}}^{2}\left({ }^{13} \mathrm{O}\right)=2.53 \pm 0.30 \mathrm{fm}^{-1}$. This value of $C_{p_{1 / 2}}^{2}\left({ }^{13} \mathrm{O}\right)$ then leads to $\mathrm{S}(0)=0.33(4) \mathrm{keV} b$ for the direct capture component. However, the resonant capture via the first excited state and the direct capture to the ground state interfere, which leads to a further increase of S-factor giving $S_{t o t}(0)=0.42(6) \mathrm{keV} \mathrm{b}$ (see Figure 13). This value is 2 orders lower than the theoretical value used by Wiescher et al. (1989). It is compatible with the analysis by Zhi-Hong (2006), who deduced the ANC from the shellmodel. Later, Timofeyuk (2013) used the source term approach using shell model wave functions and it agreed with the measured value of ANC.

Non-resonant capture dominates the ${ }^{12} \mathrm{~N}(p, \gamma){ }^{13} \mathrm{O}$ reaction at low energies, where the ANC brings important information for the R-matrix analysis.

\section{9. ${ }^{13} \mathrm{~N}(p, \gamma){ }^{14} \mathrm{O}$}

The ${ }^{13} \mathrm{~N}(\mathrm{p}, \gamma){ }^{14} \mathrm{O}$ capture is one of the important reactions in the hot CNO cycle. The rate of this reaction is dominated by the resonant capture through the first excited state of ${ }^{14} \mathrm{O}$ $\left(E_{r}=0.528 \mathrm{MeV}\right)$. The transfer reaction used in this case was ${ }^{14} \mathrm{~N}\left({ }^{13} \mathrm{~N},{ }^{14} \mathrm{O}\right){ }^{13} \mathrm{C}$ (Tang et al., 2004). The ${ }^{13} \mathrm{~N}$ radioactive beam needed for the determination of the S-factor of this capture was produced by the ${ }^{1} \mathrm{H}\left({ }^{13} \mathrm{C},{ }^{13} \mathrm{~N}\right) \mathrm{n}$ reaction (Tang et al., 2004). The ${ }^{13} \mathrm{C}$ beam of $\sim 600$ enA (electrical $\mathrm{nA}$ ) at $15 \mathrm{~A} \mathrm{MeV}$ from the K500 superconducting cyclotron bombarded a 10-cm-long,

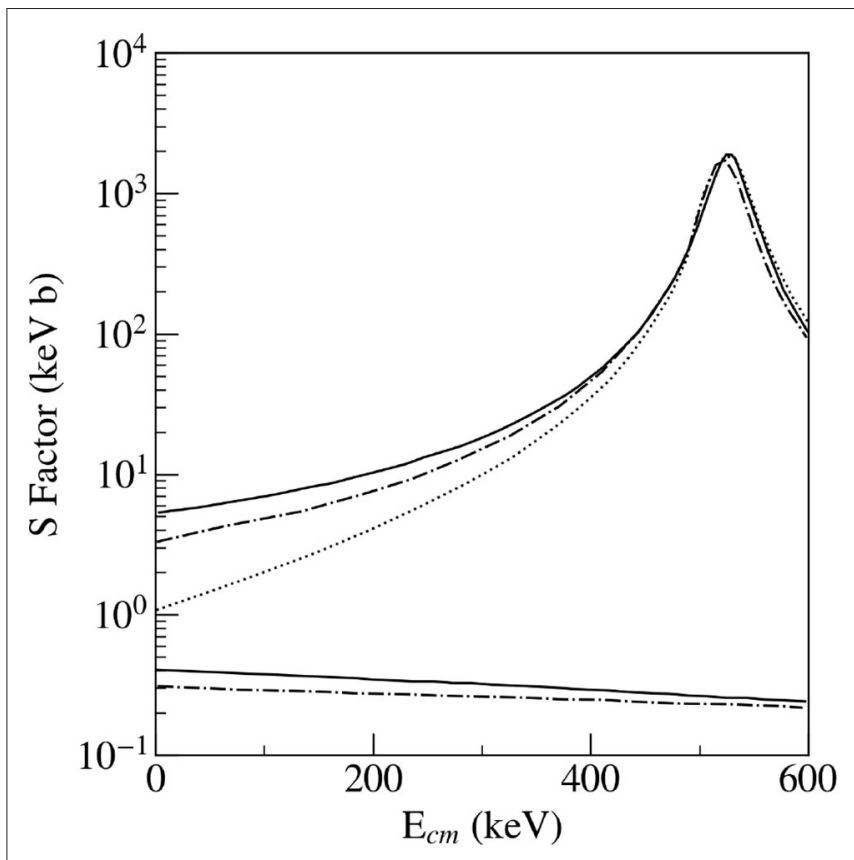

FIGURE 14 | The astrophysical S-factor for the ${ }^{13} \mathrm{~N}(\mathrm{p}, \gamma){ }^{14} \mathrm{O}$ capture. The relatively flat solid line is the direct capture contribution determined from the ANC. This result is higher than that obtained by Decrock et al. (1993) (lowest dash-dotted line). Based on constructive interference, this updated result for the total S-factor, the top solid line, is about 38\% higher than the previous result (upper dash-dotted line). For the sake of completeness, the result with destructive interference is shown (the dotted line).

$\mathrm{LN}_{2}$-cooled, cryogenic $\mathrm{H}_{2}$ gas cell with havar windows. Recoiled ${ }^{13} \mathrm{~N}$ nuclei were then separated by the magnetic spectrometer MARS at $0^{\circ}$ and focused on a target consisting of $1.5 \mathrm{mg} / \mathrm{cm}^{2}$ melamine $\mathrm{C}_{3} \mathrm{~N}_{6} \mathrm{H}_{6}$ with $20 \mu \mathrm{g} / \mathrm{cm}^{2}$ carbon backing. The reaction products from the ${ }^{13} \mathrm{~N}$ secondary beam bombarding the target were recorded by two detector telescopes, each consisted of a $5 \times 5 \mathrm{~cm} \mathrm{16-strip} \mathrm{position-sensitive} \mathrm{Si}$ detector, with a thickness of $60 \mu \mathrm{m}$, backed by a $500 \mu \mathrm{m}$ Si detector. Three sets of elastic scattering data on ${ }^{14} \mathrm{~N},{ }^{12} \mathrm{C}$, and ${ }^{1} \mathrm{H}$ were obtained.

The optical-model parameters were obtained from doublefolding-model calculations for ${ }^{13} \mathrm{~N}$ ions scattered on the melamine target (Trache et al., 2000).

After fitting the experimental angular distribution of the transfer reaction ${ }^{14} \mathrm{~N}\left({ }^{13} \mathrm{~N},{ }^{14} \mathrm{O}\right){ }^{13} \mathrm{C}$ by theoretical calculations, the value of the ANC for ${ }^{14} \mathrm{O} \rightarrow{ }^{13} \mathrm{~N}+\mathrm{p}$ was found to be $C_{p_{1 / 2}}^{2}=$ $29.0 \pm 4.3 \mathrm{fm}^{-1}$.

The S-factor was deduced from the R-matrix approach (Tang et al., 2003), where the measured ANC and the experimental resonance parameters of the (broad) first excited state was used. Parameters of Magnus et al. (1994) $\left(E_{R}^{\text {c.m. }}=527.9 \pm 1.7 \mathrm{keV}\right.$, $\gamma_{\text {total }}=37.3 \pm 0.9 \mathrm{keV}$, and $\gamma_{\gamma}=3.36 \pm 0.72 \mathrm{eV}$ ) were used for the first resonance. The total astrophysical S-factor including the constructive interference with the direct part is shown in Figure 14. This result is about $38 \%$ higher than the previous value of Decrock et al. (1993), obtained from the calculation. 
Although the applicability conditions for ANC method are not fulfilled in ${ }^{13} \mathrm{~N}(p, \gamma){ }^{14} \mathrm{O}$ reaction, the direct capture part influences the total S-factor via the interference.

\section{FURTHER USAGE OF ANCS}

The properties of ANCs have a broader potential in nuclear physics. In the next section, we briefly outline a few examples.

\subsection{Mirror Nuclei}

From theoretical calculations performed over several $0 p$ nuclei (Timofeyuk et al., 2003) it was found that the proton and neutron ANCs for mirror pairs $\left|C_{p}\right|^{2}$ and $\left|C_{n}\right|^{2}$ vary by a factor of two for different potentials used, while the ratio $R=\left|C_{p} / C_{n}\right|^{2}$ depends weakly on the used potential (less than $4 \%$ ) (Timofeyuk et al., 2003 and references therein). This effect is connected to the fact that the Coulomb potential does not vary much inside the nucleus over the nuclear volume and thus, can be replaced by a constant equal to the energy difference between the separation energies for a proton and neutron. Hence, (see Timofeyuk et al., 2003) the bound-state wave function can be written in terms of the regular Coulomb and the Bessel functions of $t^{\text {th }}$-order:

$$
\varphi_{l}(r)=\frac{F_{l}\left(i k_{p} R_{N}\right)}{k_{p} R_{N} j_{l}\left(i k_{n} R_{N}\right)} j_{l}\left(i k_{n} r\right), \quad r \leq R_{N}
$$

where $F_{l}$ is the regular Coulomb function at momentum $k_{p}$, $j_{l}$ are the Bessel functions at momentum $k_{n}$ and $R_{N}$ is the nuclear radius. $k_{p}$ and $k_{n}$ are defined by the proton and neutron separation energies. Assuming that the difference between the wave functions for mirror pairs can be neglected, the ratio will be equal to:

$$
R \approx R_{0}=\left|\frac{F_{l}\left(i k_{p} R_{N}\right)}{k_{p} R_{N} j_{l}\left(i k_{n} R_{N}\right)}\right|^{2}
$$

Considering the relation $C_{n(p)}=\sqrt{S_{n(p)}} b_{n(p)}$, where $b_{n(p)}$ is the single-particle ANC (SPANC), the equation 11 will become equal to $\left|b_{n(p)} / b_{p(n)}\right|^{2}$. Assuming that - for $p$ and $n$ - both the nuclear single-particle potentials and the single-particle wave functions in the interior are the same, the ratio will be weakly dependent from the chosen potentials.

Approaching the limits of (proton) stability, the separation energy for a neutron decreases and excited states may correspond to resonances for the proton-plus-core mirror system. In such a case, the connection between the width $\gamma_{p}$ for the resonance and the mirror ANC $C_{n}$ can be expressed (see Timofeyuk et al., 2003) as

$$
R_{\gamma}=\frac{\Gamma_{P}}{\left|C_{n}\right|^{2}} \approx \frac{k_{p}}{\mu}\left|\frac{F_{l}\left(k_{p} R_{N}\right)}{k_{p} R_{N} j_{l}\left(i k_{n} R_{N}\right)}\right|^{2}
$$

This ratio can be approximated by the single-particle ratio $R_{\gamma}^{\text {s.p. }}=\Gamma_{P}^{S . P .} / b_{n}^{2}$ if the single-particle potentials and spectroscopic factors for mirror pairs are considered equal. These relations were presented in Timofeyuk et al. (2003), where a number of mirror cases were theoretically tested, and the results were compared with the available experimental values of ANCs.

Reactions with mirror nuclei can be a solution to study reactions that involve a radioactive participant. This method was applied e.g., for ${ }^{7} \mathrm{Be}(\mathrm{p}, \gamma)^{8} \mathrm{~B}$ (Trache et al., 2003), deduced from ${ }^{8} \mathrm{Li} \rightarrow{ }^{7} \mathrm{Li}+\mathrm{n}$ using ${ }^{13} \mathrm{C}\left({ }^{7} \mathrm{Li},{ }^{8} \mathrm{Li}\right){ }^{12} \mathrm{C}$ reaction. Other cases in which the mirror nuclei procedure have been used are ${ }^{20} \mathrm{Mg}(\mathrm{p}, \gamma){ }^{21} \mathrm{Al}$ (Timofeyuk et al., 2012) from ${ }^{20} \mathrm{O}(\mathrm{n}, \gamma){ }^{21} \mathrm{O}$ using reaction $20 \mathrm{O}(\mathrm{d}, \mathrm{p}) 21 \mathrm{O},{ }^{22} \mathrm{Mg}(\mathrm{n}, \gamma)^{23} \mathrm{Mg}$ (Al-Abdullah et al., 2010) capture from ${ }^{22} \mathrm{Ne}(\mathrm{p}, \gamma){ }^{23} \mathrm{Na}$ using ${ }^{13} \mathrm{C}\left({ }^{22} \mathrm{Ne},{ }^{23} \mathrm{Ne}\right){ }^{12} \mathrm{C}$.

Using mirror reactions to study the direct $(\mathrm{p}, \gamma)$ captures proved to be an important tool, especially for radioactive nuclei. However, this technique has some potential constraints:

- Demand on peripherality of $(\mathrm{n}, \gamma)$ capture. Without the presence of Coulomb barrier the peripherality must arise from the centrifugal barrier.

- Proper treatment of $(\mathrm{d}, \mathrm{p})$ reactions, see more in a recent discussion on the role of high internal momentum of $d$ and proper application of DWBA, FR-DWBA, and CDCC (Timofeyuk et al., 2008; Gómez-Ramos and Timofeyuk, 2018).

- Mirror symmetry breaking may play a role (effect of $18 \%$ was determined for ${ }^{27} \mathrm{Mg}^{27} \mathrm{P}$ in Timofeyuk et al., 2008) and it should be treated on a case by case basis.

\subsection{Ab-initio Methods}

Recently, with the developments in ab initio methods, several techniques appeared feasible to perform ANC calculations. Despite the difficulties-convergence to long-asymptotics, sampling the tails (in Monte-Carlo methods) - the calculations can nowadays offer predictions consistent with experimental measurements for light (LiBeB) elements (Nollett and Wiringa, 2011).

\subsection{Nuclear Radii}

ANC being the normalization of the asymptotic wave function, it can naturally contain the information on nuclear halo/skin composed of the last peripheral nucleon. The possibility to access a nuclear radius, from a knowledge of overlap integrals between states with A and (A-1), was used by Timofeyuk (1998). The simple way to correlate the halo radius to ANC was used by Carstoiu et al. (2001) for the case of ${ }^{8} \mathrm{~B}$, where it was shown that the rms radius of the last proton can be determined and the proton is localized (in average) at the distance two times the core radius.

The ANC deduced from peripheral transfer reactions was used to determine the rms radii for ${ }^{12} \mathrm{~B},{ }^{13} \mathrm{C}$ by Liu et al. (2001), for ${ }^{13} \mathrm{C}$ and ${ }^{11}$ Be by Belyaeva et al. (2014), for ${ }^{12} \mathrm{~B}$ by Belyaeva et al. (2018). Rms radii of valence neutron was studied in ${ }^{16} \mathrm{~N}$ by $\mathrm{Li}$ et al. (2016) for several excited states. ${ }^{9,11} \mathrm{Be},{ }^{13} \mathrm{C}$ and alpha cluster states were studied by Ogloblin et al. (2016).

As remarked by the above authors, the determination of the rms radius by ANC brings an independent approach, that increases reliability and is a test of other methods.

\subsection{References to Computer Codes}

Different computer codes were used in the above. They are listed here with the references and web sites: 
- ECIS79 - Raynal, 1979, 1981,

- DWUCK5 - Kunz, 1990,

- FRESCO - Thompson, 1988,

- PTOLEMY - Rhoades-Brown et al., 1983,

- RADCAP - Bertulani, 2003

\section{SUMMARY}

The ANC method is used for determination of the astrophysical $\mathrm{S}$-factor at low astrophysical energies of participating particles, where the cross-section is very low, and it is extremely difficult to measure it directly. Where the experimental effort is challenging and the case is favorable, mirror symmetry is/can be used to deduce the ANCs of bound states or properties of resonances.

We have illustrated the usefulness, but also limitations of the indirect ANC method in several examples with an astrophysical interest. They include experiments with solid or isotopic gas targets with stable beams and also experiments in inverse kinematics with radioactive beams.

The ANC method was successfully tested in number of reactions, found compatible with theory predictions as shown in the summarizing (Table 2). ANCs are also frequently deduced from R-matrix fits. These values are also generally in agreement with independently found ANCs.

An important region of ANC method application are measurements with radioactive beams. The reactions with the short-lived isotopes are still not accessible to direct measurements. Nuclei distant from the valley of stability have lower separation energies and thus the level density around threshold may be smaller. This is favorable to the direct radiative

\section{REFERENCES}

Abia, C., Hedrosa, R. P., Domínguez, I., and Straniero, O. (2017). The puzzle of the $\mathrm{CNO}$ isotope ratios in asymptotic giant branch carbon stars. Astron. Astrophys. 599:A39. doi: 10.1051/0004-6361/201629969

Ahmad, Q. R., Allen, R. C., Andersen, T. C., Anglin, J. D., Bühler, G., Barton, J. C., et al. (2001). Measurement of the rate of $v_{e}+d \rightarrow p+p+e^{-}$interactions produced by ${ }^{8} \mathrm{~B}$ solar neutrinos at the sudbury neutrino observatory. Phys. Rev. Lett. 87:071301. doi: 10.1103/PhysRevLett.87.071301

Al-Abdullah, T., Carstoiu, F., Chen, X., Clark, H. L., Fu, C., Gagliardi, C. A., et al. (2010). Stellar reaction rate for ${ }^{22} \mathrm{Mg}+\mathrm{p} \rightarrow{ }^{23} \mathrm{Al}$ from the asymptotic normalization coefficient in the mirror nuclear system ${ }^{22} \mathrm{Ne}+\mathrm{n} \rightarrow{ }^{23} \mathrm{Ne}$. Phys. Rev. C 81:035802. doi: 10.1103/PhysRevC.81.035802

Azhari, A., Burjan, V., Carstoiu, F., Dejbakhsh, H., Gagliardi, C. A., Kroha, V., et al. (1999). The ${ }^{10} \mathrm{~B}\left({ }^{7} \mathrm{Be},{ }^{8} \mathrm{~B}\right){ }^{9} \mathrm{Be}$ reaction and the ${ }^{7} \mathrm{Be}(\mathrm{p}, \gamma){ }^{8} \mathrm{~B} \mathrm{~S}$ factor. Phys. Rev. Lett. 82, 3960-3963.

Azhari, A., Burjan, V., Carstoiu, F., Gagliardi, C. A., Kroha, V., Mukhamedzhanov, A. M., et al. (2001). Asymptotic normalization coefficients and the ${ }^{7} \mathrm{Be}(\mathrm{p}, \gamma){ }^{8} \mathrm{~B}$ astrophysical S factor. Phys. Rev. C 63:055803. doi: 10.1103/PhysRevC.63.055803

Bahcall, J. N. (1985). The solar neutrino problem. Solar Phys. 100, 53-63.

Banu, A., Al-Abdullah, T., Fu, C., Gagliardi, C. A., McCleskey, M., Mukhamedzhanov, A. M., et al. (2009). Astrophysical S factor for the radiative capture ${ }^{12} \mathrm{~N}(p, \gamma){ }^{13} \mathrm{O}$ determined from the ${ }^{14} \mathrm{~N}\left({ }^{12} \mathrm{~N},{ }^{13} \mathrm{O}\right){ }^{13} \mathrm{C}$ proton transfer reaction. Phys. Rev. C 79:025805. doi: 10.1103/PhysRevC.79.025805

Baur, G., Bertulani, C. A., and Rebel, H. (1986). Coulomb dissociation as a source of information on radiative capture processes of astrophysical interest. Nuclear Phys. A 458, 188-204.

Baur, G., and Rebel, H. (1996). Coulomb breakup of nuclei-applications to Astrophysics. Annu. Rev. Nuclear Part. Sci. 46, 321-350. capture contribution to the total astrophysical S-factor. Reactions with radioactive beams, however, always need more development both in the experimental techniques (quality of elastic scattering data, resolutions) and in theoretical analysis (reliable OMP, reaction codes). This is discussed in more detail in the remarks to $\mathrm{ECT}^{\star}$ workshop by Trache and Carstoiu (2019).

\section{AUTHOR CONTRIBUTIONS}

All the listed authors contributed to the writing of the paper. $\mathrm{VB}$ worked on all experiments in section 3, JM has worked on ${ }^{14} \mathrm{C}(\mathrm{n}, \gamma){ }^{15} \mathrm{C},{ }^{15} \mathrm{~N}(\mathrm{p}, \gamma){ }^{16} \mathrm{O}$, and ${ }^{18} \mathrm{O}(\mathrm{p}, \gamma){ }^{19} \mathrm{~F}$.

\section{FUNDING}

We would like to thank for the support to the MEYS project SPIRAL2-CZ, EF16-013/0001679.

\section{ACKNOWLEDGMENTS}

Authors want to thank to all our colleagues from the Cyclotron Institute, Texas A\&M University, College Station, Texas, colleagues from Istituto Nazionale di Fisica Nucleare, Laboratori Nazionali del Sud, Italia, Sicilia, Catania, and colleagues from the Nuclear Physics Institute of the Czech Academy of Sciences with whom we were happy to collaborate on topics included in this review. Authors are also indebted to referees, whose insightful suggestions and comments importantly improved the original text. The figures are adapted from the papers referred in each reaction.

Bellerive, A., Klein, J. R., McDonald, A. B., Noble, A. J., and Poon, A. W. P. (2016). The Sudbury Neutrino observatory. Nuclear Phys. B 908, 30-51. doi: 10.1016/j.nuclphysb.2016.04.035

Belyaeva, T. L., Goncharov, S. A., Demyanova, A. S., Ogloblin, A. A., Danilov, A. N., Maslov, V. A., et al. (2018). Neutron halos in the excited states of ${ }^{12}$ B. Phys. Rev. C 98:034602. doi: 10.1103/PhysRevC.98.034602

Belyaeva, T. L., Perez-Torres, R., Ogloblin, A. A., Demyanova, A. S., Ershov, S. N., and Goncharov, S. A. (2014). Determination of neutron halo radii in the first excited states of ${ }^{13} \mathrm{C}$ and ${ }^{11} \mathrm{Be}$ with the asymptotic normalization coefficients method. Phys. Rev. C 90:064610. doi: 10.1103/PhysRevC.90. 064610

Bém, P., Burjan, V., Kroha, V., Novák, J., Piskor, S., Šimečková, E., et al. (2000). Asymptotic normalization coefficients for ${ }^{14} \mathrm{~N} \leftrightarrow{ }^{13} \mathrm{C}+p$ from ${ }^{13} \mathrm{C}\left({ }^{3} \mathrm{He}, d\right){ }^{14} \mathrm{~N}$. Phys. Rev. C 62:024320. doi: 10.1103/PhysRevC.62.024320

Bertulani, C. A. (2003). RADCAP: A potential model tool for direct capture reactions. Comput. Phys. Commun. 156, 123-141. doi: 10.1016/S0010-4655(03)00441-7

Bing, G., and Zhi-Hong, L. (2007). Determination of astrophysical ${ }^{13} \mathrm{~N}(\mathrm{p}, \gamma){ }^{14} \mathrm{O}$ $\mathrm{S}$-factors from the asymptotic normalization coefficient of ${ }^{14} \mathrm{C} \rightarrow{ }^{13} \mathrm{C}+\mathrm{n}$. Chin. Phys. Lett. 24, 65-68. doi: 10.1088/0256-307X/24/1/018

Buckner, M. Q., Iliadis, C., Cesaratto, J. M., Howard, C., Clegg, T. B., Champagne, A. E., et al. (2012). Thermonuclear reaction rate of ${ }^{18} \mathrm{O}(\mathrm{p}, \gamma)^{19}$ F. Phys. Rev. $C$ 86:065804. doi: 10.1103/PhysRevC.86.065804

Burjan, V., Hons, Z., Kroha, V., Mrázek, J., Piskor, S., Mukhamedzhanov, A. M., et al. (2013). Experimental study of the ${ }^{18} \mathrm{O}(\mathrm{d}, \mathrm{p}){ }^{19} \mathrm{O}$ reaction and the ANC method. J. Phys. Conf. Ser. 420:012142. doi: 10.1088/1742-6596/420/1/ 012142

Burjan, V., Hons, Z., Kroha, V., Mrázek, J., Piskor, S., Mukhamedzhanov, A. M., et al. (2014). The ${ }^{18} \mathrm{O}(\mathrm{d}, \mathrm{p}){ }^{19} \mathrm{O}$ reaction and the ANC method. AIP Conf. Proc. 1595, 163-167. doi: 10.1063/1.4875304 
Burjan, V., Hons, Z., Kroha, V., Mrazek, J., Piskor, S., Mukhamedzhanov, A. M., et al. (2019). The determination of the astrophysical S-factor of the direct ${ }^{18} \mathrm{O}(\mathrm{p}, \gamma){ }^{19} \mathrm{~F}$ capture by the ANC method. Eur. Phys. J. A 55:114. doi: 10.1140/epja/i2019-12801-8

Carstoiu, F., Trache, L., Gagliardi, C. A., Tribble, R. E., and Mukhamedzhanov, A. M. (2001). Radius of ${ }^{8} \mathrm{~B}$ halo from the asymptotic normalization coefficient. Phys. Rev. C 63:054310. doi: 10.1103/PhysRevC.63.054310

Chow, H. C., Griffiths, G. M., and Hall, T. H. (1975). The ${ }^{16} \mathrm{O}(\mathrm{p}, \gamma){ }^{17} \mathrm{~F}$ direct capture cross section with an extrapolation to astrophysical energies. Can. J. Phys. 53, 1672-1686. doi: 10.1139/p75-213

Daehnick, W. W., Childs, J. D., and Vrcelj, Z. (1980). Global optical model potential for elastic deuteron scattering from 12 to $90 \mathrm{MeV}$. Phys. Rev. C 21, 2253-2274. doi: 10.1103/PhysRevC.21.2253

Decrock, P., Gaelens, M., Huyse, M., Reusen, G., Vancraeynest, G., Van Duppen, P., et al. (1993). Study of the ${ }^{13} \mathrm{~N}(d, n){ }^{14} \mathrm{O}$ reaction cross section and its astrophysical implications for the ${ }^{13} \mathrm{~N}$ proton capture reaction. Phys. Rev. C 48, 2057-2067. doi: 10.1103/PhysRevC.48.2057

Duke, C. B. (1963). Optical-model analysis of elastic scattering of protons on oxygen at intermediate energies. Phys. Rev. 129, 681-691. doi: 10.1103/PhysRev.129.681

Gagliardi, C., Azhari, A., Burjan, V., Carstoiu, F., Kroha, V., Mukhamedzhanov, A., et al. (2002). Asymptotic normalization coefficients and astrophysical direct capture rates. Eur. Phys. J. A 13, 227-232. doi: 10.1140/epja1339-38

Gagliardi, C. A., Azhari, A., Bem, P., Burjan, V., Carstoiu, F., Cejpek, J., et al. (2001). Asymptotic normalization coefficients from direct transfer reactions and astrophysical S factors. Nuclear Phys. A 682, 369-374. doi: 10.1016/S0375-9474(00)00662-X

Gagliardi, C. A., Tribble, R. E., Azhari, A., Clark, H. L., Lui, Y.-W., Mukhamedzhanov, A. M., et al. (1999). Tests of transfer reaction determinations of astrophysical S factors. Phys. Rev. C 59, 1149-1153. doi: 10.1103/PhysRevC.59.1149

Gómez-Ramos, M., and Timofeyuk, N. K. (2018). Reduced sensitivity of the $(d, p)$ cross sections to the deuteron model beyond the adiabatic approximation. Phys. Rev. C 98:011601. doi: 10.1103/PhysRevC.98.011601

Guo, B., Su, J., Li, Z. H., Wang, Y. B., Yan, S. Q., Li, Y. J., et al. (2013). Determination of the astrophysical ${ }^{12} \mathrm{~N}(\mathrm{p}, \gamma)^{13} \mathrm{O}$ reaction rate from the ${ }^{2} \mathrm{H}\left({ }^{12} \mathrm{~N},{ }^{13} \mathrm{O}\right) \mathrm{n}$ reaction and its astrophysical implications. Phys. Rev. C 87:015803. doi: 10.1103/PhysRevC.87.015803

Herndl, H., Hofinger, R., Jank, J., Oberhummer, H., Görres, J., Wiescher, M., et al. (1999). Reaction rates for neutron capture reactions to C, N, and $\mathrm{O}$ isotopes to the neutron rich side of stability. Phys. Rev. C 60:064614. doi: 10.1103/PhysRevC.60.064614

Huang, J. T., Bertulani, C. A., and Guimarães, V. (2010). Radiative capture of nucleons at astrophysical energies with single-particle states. Atom Data Nuclear Data Tables 96, 824-847. doi: 10.1016/j.adt.2010.06.004

Imai, N., Aoi, N., Kubono, S., Beaumel, D., Abe, K., Kato, S., et al. (2001). Test of the ANC method via (d,p) reaction. Nuclear Phys. A 688, 281-284. doi: 10.1016/S0375-9474(01)00715-1

Jeukenne, J.-P., Lejeune, A., and Mahaux, C. (1977). Optical-model potential in finite nuclei from Reid's hard core interaction. Phys. Rev. C 16, 80-96. doi: $10.1103 /$ PhysRevC.16.80

Kunz, P. (1990). DWUCK5: computer code DWUCK5: distorted wave Born approximation. Available online at: http://www.oecd-nea.org/tools/abstract/ detail/nesc9872/

Lee, D. W., Powell, J., Perajarvi, K., Guo, F. Q., Moltz, D. M., and Cerny, J. (2011). Study of the ${ }^{11} \mathrm{C}(\mathrm{p}, \gamma)$ reaction via the indirect $\mathrm{d}\left({ }^{11} \mathrm{C},{ }^{12} \mathrm{~N}\right) \mathrm{n}$ transfer reaction. J. Phys. G Nuclear Part. Phys. 38:075201. doi: 10.1088/0954-3899/38/7/075201

Lefebvre, A., Aguer, P., Kiener, J., Bogaert, G., Coc, A., de Oliveira, F., et al. (1995). Astrophysical rate of the ${ }^{11} \mathrm{C}+\mathrm{p}$ reaction from the coulomb break-up of a ${ }^{12} \mathrm{~N}$ radioactive beam. Nuclear Phys. A 592, 69-88. doi: 10.1016/0375-9474(95)00291-8

Li, E. T., Guo, B., Li, Z. H., Wang, Y. B., Li, Y. J., Wu, Z. D., et al. (2016). Radii of the bound states in ${ }^{16} \mathrm{~N}$ from the asymptotic normalization coefficients. Chin. Phys. C 40:114104. doi: 10.1088/1674-1137/40/11/114104

Liu, Z. H., Lin, C. J., Zhang, H. Q., Li, Z. C., Zhang, J. S., Wu, Y. W., et al. (2001). Asymptotic normalization coefficients and neutron halo of the excited states in ${ }^{12} \mathrm{~B}$ and ${ }^{13} \mathrm{C}$. Phys. Rev. C 64:034312. doi: 10.1103/PhysRevC.64. 034312
Lorenz-Wirzba, H., Schmalbrock, P., Trautvetter, H. P., Wiescher, M., Rolfs, C., and Rodney, W. S. (1979). The ${ }^{18} \mathrm{O}(\mathrm{p}, \alpha)^{15} \mathrm{~N}$ reaction at stellar energies. Nuclear Phys. A 313, 346-362. doi: 10.1016/0375-9474(79)90505-0

Magnus, P. V., Adelberger, E. G., and Garcia, A. (1994). The "hot CNO cycle" ${ }^{13} \mathrm{~N}(p, \gamma)$ resonance energy and the ${ }^{18} \mathrm{Ne}$ mass. Phys. Rev. C 49, R1755-R1758. doi: 10.1103/PhysRevC.49.R1755

Meissner, J., Schatz, H., Görres, J., Herndl, H., Wiescher, M., Beer, H., et al. (1996). Neutron capture cross section of ${ }^{18} \mathrm{O}$ and its astrophysical implications. Phys. Rev. C 53, 459-468. doi: 10.1103/PhysRevC.53.459

Morlock, R., Kunz, R., Mayer, A., Jaeger, M., Müller, A., Hammer, J. W., et al. (1997). Halo properties of the first $1 / 2^{+}$state in ${ }^{17} \mathrm{~F}$ from the ${ }^{16} \mathrm{O}(\mathrm{p}, \gamma){ }^{17} \mathrm{~F}$ reaction. Phys. Rev. Lett. 79, 3837-3840. doi: 10.1103/PhysRevLett.79.3837

Mukhamedzhanov, A., Burjan, V., Gulino, M., Hons, Z., Kroha, V., McCleskey, M., et al. (2011). Asymptotic normalization coefficients from the ${ }^{14} \mathrm{C}(\mathrm{d}, \mathrm{p})^{15} \mathrm{C}$ reaction. Phys. Rev. C 84:024616. doi: 10.1103/PhysRevC.84.024616

Mukhamedzhanov, A. M., Bém, P., Brown, B. A., Burjan, V., Gagliardi, C. A., Kroha, V., et al. (2003). Asymptotic normalization coefficients for ${ }^{14} \mathrm{~N}+p \rightarrow{ }^{15}$ $\mathrm{O}$ and the astrophysical S factor for ${ }^{14} \mathrm{~N}(p, \gamma)^{15} \mathrm{O}$. Phys. Rev. C 67:065804. doi: 10.1103/PhysRevC.68.019901

Mukhamedzhanov, A. M., Bém, P., Burjan, V., Gagliardi, C. A., Goldberg, V. Z. Hons, Z., et al. (2008). New astrophysical S factor for the ${ }^{15} \mathrm{~N}(p, \gamma)^{16} \mathrm{O}$ reaction via the asymptotic normalization coefficient (ANC) method. Phys. Rev. C 78:015804. doi: 10.1103/PhysRevC.78.015804

Mukhamedzhanov, A. M., Bém, P., Burjan, V., Gagliardi, C. A., Irgaziev, B. F., Kroha, V., et al. (2006). Asymptotic normalization coefficients from the ${ }^{20} \mathrm{Ne}\left({ }^{3} \mathrm{He}, \mathrm{d}\right){ }^{21} \mathrm{Na}$ reaction and astrophysical factor for ${ }^{20} \mathrm{Ne}(p, \gamma)^{21} \mathrm{Na}$. Phys. Rev. C 73:035806. doi: 10.1103/PhysRevC.73.035806

Mukhamedzhanov, A. M., Clark, H. L., Gagliardi, C. A., Lui, Y.-W., Trache, L., Tribble, R. E., et al. (1997). Asymptotic normalization coefficients for ${ }^{10} \mathrm{~B} \rightarrow{ }^{9} \mathrm{Be}+$ p. Phys. Rev. C 56, 1302-1312. doi: 10.1103/PhysRevC. 56.1302

Mukhamedzhanov, A. M., Tribble, R. E., and Timofeyuk, N. K. (1995). Possibility to determine the astrophysical $\mathrm{S}$ factor for the ${ }^{7} \mathrm{Be}(\mathrm{p}, \gamma){ }^{8} \mathrm{~B}$ radiative capture from analysis of the ${ }^{7} \mathrm{Be}\left({ }^{3} \mathrm{He}, \mathrm{d}\right){ }^{8} \mathrm{~B}$ reaction. Phys. Rev. C 51, 3472-3478. doi: 10.1103/PhysRevC.51.3472

Nollett, K. M., and Wiringa, R. B. (2011). Asymptotic normalization coefficients from ab initio calculations. Phys. Rev. C 83:041001. doi: 10.1103/PhysRevC.83.041001

Ogloblin, A. A., Demyanova, A. S., Danilov, A. N., Belyaeva, T. L., Goncharov, S. A., and Trzaska, W. (2016). Nuclear states with anomalously large radius (size isomers). Phys. Atom. Nuclei 79, 514-524. doi: 10.1134/S1063778816040177

Ohsaki, T., Igashira, M., Nagai, Y., Segawa, M., and Muto, K. (2008). Role of multiparticle-multihole states of ${ }^{18,19} \mathrm{O}$ in ${ }^{18} \mathrm{O}(n, \gamma){ }^{19} \mathrm{O}$ reactions at $\mathrm{keV}$ energy. Phys. Rev. C 77:051303. doi: 10.1103/PhysRevC.77.051303

Palmerini, S., Trippella, O., and Busso, M. (2017). A deep mixing solution to the aluminum and oxygen isotope puzzles in pre-solar grains. Monthly Notice $R$ Astron. Soc. 467, 1193-1201. doi: 10.1093/mnras/stx137

Perey, C. M., and Perey, F. G. (1976). Compilation of phenomenological opticalmodel parameters 1954-1975. Atomic Data Nuclear Data Tables 17, 1-101. doi: 10.1016/0092-640X(76)90007-3

Raynal, J. (1979). Code ECIS79. Available online at: https://www.oecd-nea.org/ tools/abstract/detail/nea-0850; https://people.nscl.msu.edu/ brown/reactioncodes/

Raynal, J. (1981). Recurrence relations for distorted-wave Born approximation Coulomb excitation integrals and their use in coupled channel calculations. Phys. Rev. C 23, 2571-2585. doi: 10.1103/PhysRevC.23.2571

Rhoades-Brown M., Steven, P., and McFarlane M. (1983). Ptolemy: a program for heavy-ion direct-reaction calculations. Available online at: https://inis.iaea.org/ search/searchsinglerecord.aspx?recordsFor=SingleRecord\&RN=9416330

Rolfs, C., Rodney, W. S., Shapiro, M. H., and Winkler, H. (1975). Hydrogen burning of ${ }^{20} \mathrm{Ne}$ and ${ }^{22} \mathrm{Ne}$ in stars. Nuclear Phys. A 241, 460-486. doi: 10.1016/0375-9474(75)90398-X

Rolfs, C. E., and Rodney, W. S. (1988). Cauldrons in the Cosmos: Nuclear Astrophysics. Chicago, IL: University of Chicago Press.

Tang, X., Azhari, A., Fu, C., Gagliardi, C. A., Mukhamedzhanov, A. M., Pirlepesov, F., et al. (2004). Determination of the direct capture contribution for ${ }^{13} \mathrm{~N}(p, \gamma){ }^{14} \mathrm{O}$ from the ${ }^{14} \mathrm{O} \rightarrow{ }^{13} \mathrm{~N}+p$ asymptotic normalization coefficient. Phys. Rev. C 69:055807. doi: 10.1103/PhysRevC.69.055807 
Tang, X., Azhari, A., Gagliardi, C. A., Mukhamedzhanov, A. M., Pirlepesov, F., Trache, L., et al. (2003). Determination of the astrophysical S factor for ${ }^{11} \mathrm{C}(p, \gamma){ }^{12} \mathrm{~N}$ from the ${ }^{12} \mathrm{~N} \rightarrow{ }^{11} \mathrm{C}+p$ asymptotic normalization coefficient. Phys. Rev. C 67:015804. doi: 10.1103/PhysRevC.67.015804

Thompson, I. J. (1988). Coupled reaction channels calculations in nuclear physics. Comp. Phys. Rep. 7, 167-212. doi: 10.1016/0167-7977(88)90005-6

Timofeyuk, N. K. (1998). One nucleon overlap integrals for light nuclei. Nuclear Phys. A 632, 19-38. doi: 10.1016/S0375-9474(98)00810-0

Timofeyuk, N. K. (2010). Overlap functions, spectroscopic factors, and asymptotic normalization coefficients generated by a shell-model source term. Phys. Rev. C 81:064306. doi: 10.1103/PhysRevC.81.064306

Timofeyuk, N. K. (2013). Spectroscopic factors and asymptotic normalization coefficients for 0p-shell nuclei: recent updates. Phys. Rev. C 88:044315. doi: 10.1103/PhysRevC.88.044315

Timofeyuk, N. K., Descouvemont, P., and Thompson, I. J. (2008). Threshold effects in the ${ }^{27} \mathrm{P}\left(\frac{3}{2}^{+}\right) \rightarrow{ }^{26} \mathrm{Si}+p$ and ${ }^{27} \mathrm{Mg}\left(\frac{3}{2}^{+}\right) \rightarrow{ }^{26} \mathrm{Mg}+n$ mirror decays and the stellar reaction ${ }^{26} \mathrm{Si}(p, \gamma){ }^{27} \mathrm{P}$. Phys. Rev. C 78:044323. doi: 10.1103/PhysRevC.78.044323

Timofeyuk, N. K., Fernández-Domínguez, B., Descouvemont, P., Catford, W. N., Delaunay, F., and Thomas, J. S. (2012). Core excitations and narrow states beyond the proton dripline: the exotic nucleus ${ }^{21}$ Al. Phys. Rev. C 86:034305. doi: 10.1103/PhysRevC.86.034305

Timofeyuk, N. K., Johnson, R. C., and Mukhamedzhanov, A. M. (2003). Relation between proton and neutron asymptotic normalization coefficients for light mirror nuclei and its relevance to nuclear astrophysics. Phys. Rev. Lett. 91:232501. doi: 10.1103/PhysRevLett.91.232501

Trache, L., Azhari, A., Carstoiu, F., Clark, H. L., Gagliardi, C. A., Lui, Y.-W., et al. (2003). Asymptotic normalization coefficients for ${ }^{8} \mathrm{~B} \rightarrow{ }^{7} \mathrm{Be}+p$ from a study of ${ }^{8} \mathrm{Li} \rightarrow{ }^{7} \mathrm{Li}+$ n. Phys. Rev. C 67:062801. doi: 10.1103/PhysRevC.67.062801

Trache, L., Azhari, A., Clark, H. L., Gagliardi, C. A., Lui, Y.-W., Mukhamedzhanov, A. M., et al. (1998). Asymptotic normalization coefficients for ${ }^{13} \mathrm{C}+p \rightarrow{ }^{14} \mathrm{~N}$. Phys. Rev. C 58, 2715-2719. doi: 10.1103/PhysRevC.58.2715

Trache, L., Azhari, A., Clark, H. L., Gagliardi, C. A., Lui, Y.-W., Mukhamedzhanov, A. M., et al. (2000). Optical model potentials involving loosely bound p-shell nuclei around $10 \mathrm{MeV} /$ nucleon. Phys. Rev. C 61:024612. doi: 10.1103/PhysRevC.61.024612

Trache, L., and Carstoiu, F. (2019). Indirect methods in nuclear astrophysics. arXiv preprint arXiv:1911.06077. Available online at: http://arxiv.org/abs/1911.06077

Vaughn, F. J., Grench, H. A., Imhof, W. L., Rowland, J. H., and Walt, M. (1965). The total neutron cross section of O18 from 0.14 to
2.47 MeV. Nuclear Phys. 64, 336-342. doi: 10.1016/0029-5582(65) 90361-5

Vernotte, J., Berrier-Ronsin, G., Kalifa, J., Tamisier, R., and Wildenthal, B. H. (1994). Spectroscopic factors from one-proton stripping reactions on sdshell nuclei: Experimental measurements and shell-model calculations. Nuclear Phys. A 571, 1-42. doi: 10.1016/0375-9474(94)90339-5

Watson, B. A., Singh, P. P., and Segel, R. E. (1969). Optical-model analysis of nucleon scattering from 1 p-shell nuclei between 10 and 50 meV. Phys. Rev. 182, 977-989. doi: 10.1103/PhysRev.182.977

Wiescher, M., Becker, H. W., Görres, J., Kettner, K. U., Trautvetter, H. P., Kieser, W. E., et al. (1980). Nuclear and astrophysical aspects of $18 \mathrm{O}(\mathrm{p}, \gamma) 19 \mathrm{~F}$. Nuclear Phys. A 349, 165-216. doi: 10.1016/0375-9474(80)90451-0

Wiescher, M., Gorres, J., Graff, S., Buchmann, L., and Thielemann, F.-K. (1989). The hot proton-proton chains in low-metallicity objects. Astrophys. J. 343, 352-364. doi: 10.1086/167709

Wiescher, M., Gorres, J., and Thielemann, F.-K. (1990). Capture reactions on C-14 in nonstandard big bang nucleosynthesis. Astrophys. J. 363, 340-343. doi: $10.1086 / 169348$

Xu, H. M., Gagliardi, C. A., Tribble, R. E., Mukhamedzhanov, A. M., and Timofeyuk, N. K. (1994). Overall normalization of the astrophysical S factor and the nuclear vertex constant for ${ }^{7} \mathrm{Be}(p, \gamma)^{8} \mathrm{~B}$ reactions. Phys. Rev. Lett. 73, 2027-2030. doi: 10.1103/PhysRevLett.73.2027

Yarmukhamedov, R., and Blokhintsev, L. D. (2018). Asymptotic normalization coefficients (nuclear vertex constants), three-body asymptotic normalization functions (on-shell vertex functions) and nuclear astrophysics. Phys. Atom. Nuclei 81, 616-629. doi: 10.1134/\$1063778818050198

Zhi-Hong, L. (2006). Astrophysical rates for ${ }^{12} \mathrm{~N}(p, \gamma)^{13} \mathrm{O}$ direct capture reaction. Chin. Phys. Lett. 23, 3219-3221. doi: 10.1088/0256-307X/23/12/027

Conflict of Interest: The authors declare that the research was conducted in the absence of any commercial or financial relationships that could be construed as a potential conflict of interest.

Copyright (c) 2020 Burjan, Mrazek and D'Agata. This is an open-access article distributed under the terms of the Creative Commons Attribution License (CC BY). The use, distribution or reproduction in other forums is permitted, provided the original author(s) and the copyright owner(s) are credited and that the original publication in this journal is cited, in accordance with accepted academic practice. No use, distribution or reproduction is permitted which does not comply with these terms. 